\title{
Comprehensive atmospheric modeling of reactive cyclic siloxanes and their oxidation products
}

Nathan J. Janechek et al.

Correspondence to: Charles O. Stanier (charles-stanier@uiowa.edu)

The copyright of individual parts of the supplement might differ from the CC BY 3.0 License. 


\section{Table of Contents}

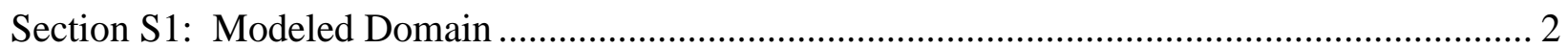

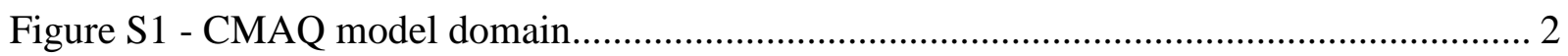

Section S2: Deposition Sensitivity .............................................................................. 3

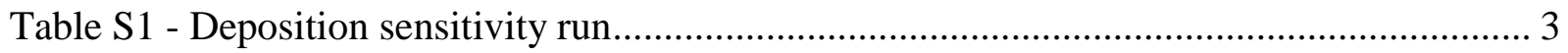

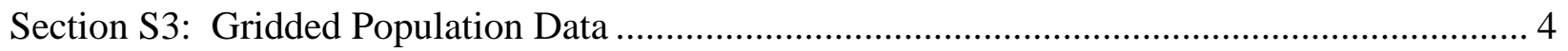

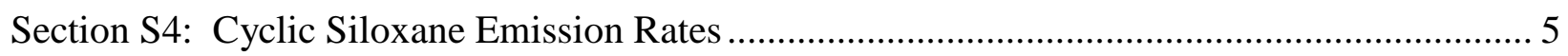

Table S2 - Cyclic siloxane emission rates ....................................................................... 5

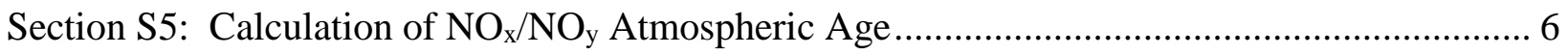

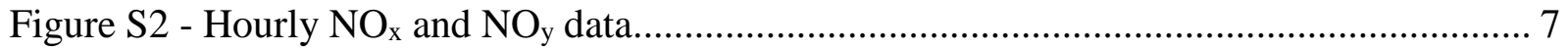

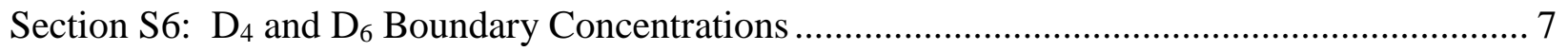

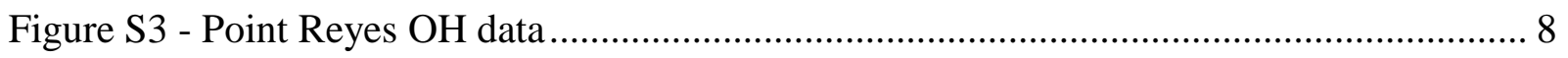

Figure S4 - Monthly dependent $\mathrm{D}_{4} / \mathrm{D}_{5}$ and $\mathrm{D}_{6} / \mathrm{D}_{5}$ boundary ratios ..................................... 9

Section S7: Analyzed Sites ......................................................................................... 10

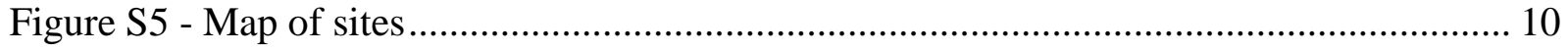

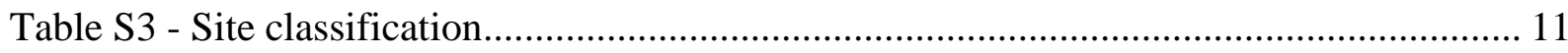

Table S4 - Cyclic siloxane modeled surface concentrations .................................................. 12

Table S5 - Oxidized cyclic siloxane modeled surface concentrations...................................... 13

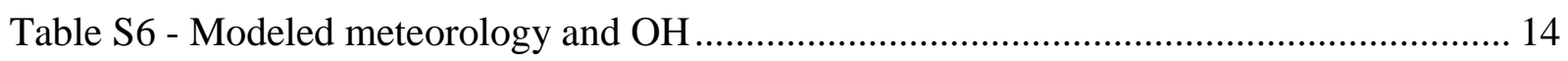

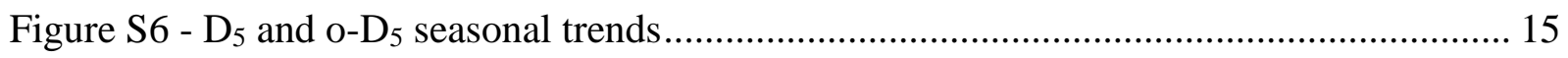

Table S7 - Modeled compound ratios ........................................................................... 16

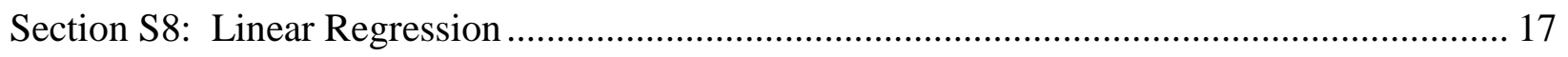

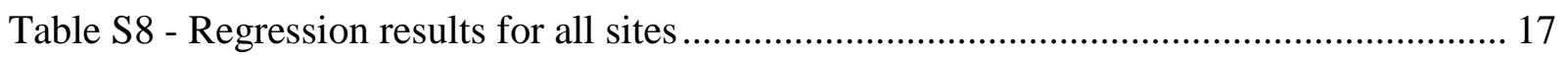

Table S9 - Regression results excluding Canadian and Point Reyes sites............................... 18

Figure S7 - PBL·WS regression fit ................................................................................ 19

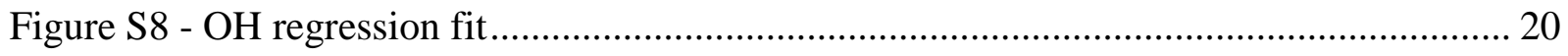

Section S9: Midwest Model Performance ................................................................................. 21

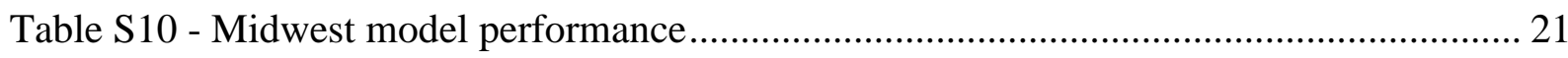

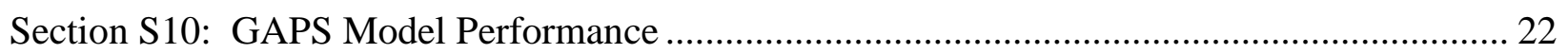

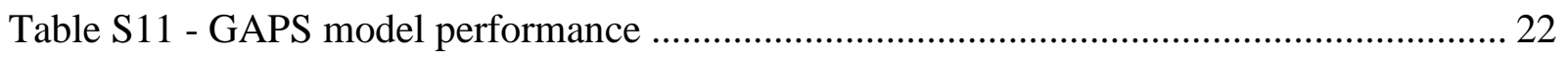

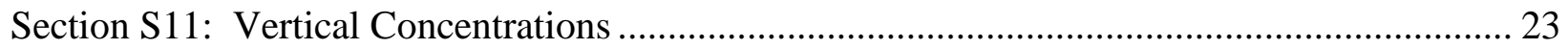

Figure S9 - Analyzed grid cells ............................................................................... 23

Figure S10 - Modeled vertical OH concentrations ………………………………………..... 24 


\section{Section S1： Modeled Domain}

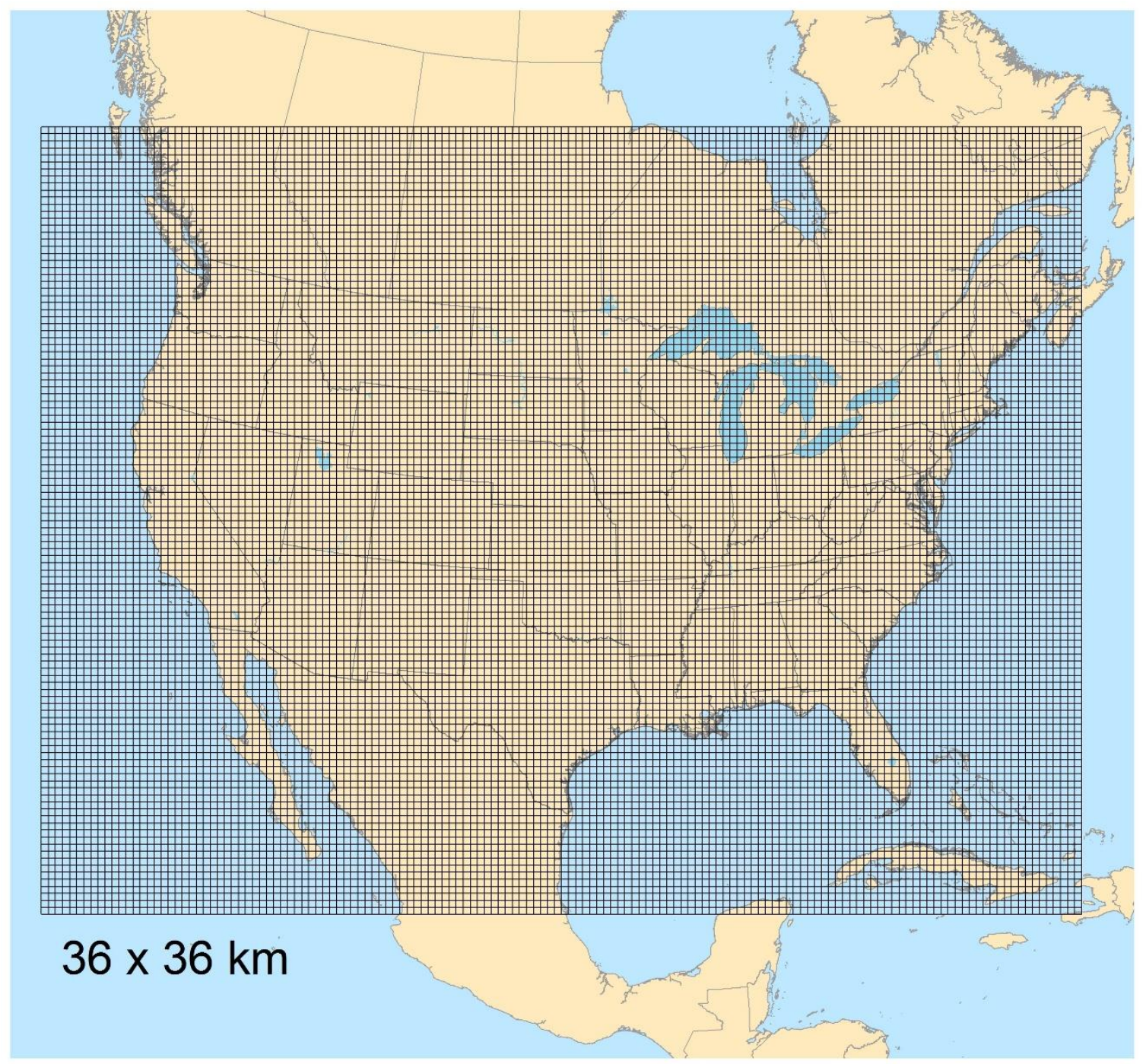

Figure S1: CMAQ model domain. 


\section{Section S2: Deposition Sensitivity}

A wet and dry deposition sensitivity test compared gas phase concentrations with and without deposition as a check to verify cVMS deposition parameters. CMAQ includes treatment of dry and wet deposition (Byun et al., 1999; Roselle and Binkowski, 1999) parameterized by Henry's law coefficients, mass diffusivity, reactivity relative to $\mathrm{HNO}_{3}$, and mesophyll resistance. However, there is considerable uncertainty in these cVMS parameterizations and only the $\mathrm{D}_{4}, \mathrm{D}_{5}$, and $\mathrm{D}_{6}$ Henry's law coefficients (Xu and Kropscott, 2012) have been experimentally determined. A 1-week sensitivity analysis for a Chicago grid cell over the period of August 13 - August 20, 2004 was run for three scenarios 1) no deposition, 2) dry deposition only, and 3) dry and wet deposition. The addition of deposition caused gas phase concentrations to decrease, the percent change is displayed in Table S1. Wet deposition for the parent compounds was not observed but the single $\mathrm{OH}$ substituted oxidized species did undergo wet deposition, albeit small. Similarly, for dry deposition the parent compounds underwent less deposition than the oxidized species. Modeled wet and dry deposition agree with expected behavior based on the input parameters and the current understanding that as cVMS species are oxidized, deposition increases (Whelan et al., 2004).

Table S1: Deposition sensitivity test. August 13-20, 2004 average mixing ratios for Chicago grid cell.

\begin{tabular}{|c|c|c|c|c|c|}
\hline & $\begin{array}{c}\text { No Deposition } \\
(\mathrm{ppm})\end{array}$ & $\begin{array}{c}\text { Dry Deposition } \\
(\mathrm{ppm})\end{array}$ & $\begin{array}{c}\text { Dry and Wet } \\
\text { Deposition (ppm) }\end{array}$ & $\begin{array}{c}\text { Percent Change } \\
\text { due to Dry } \\
\text { Deposition (\%) }\end{array}$ & $\begin{array}{c}\text { Percent Change } \\
\text { due to Wet } \\
\text { Deposition (\%) }\end{array}$ \\
\hline $\mathrm{D}_{4}$ & $6.16 \mathrm{E}-06$ & $5.86 \mathrm{E}-06$ & $5.86 \mathrm{E}-06$ & 4.86 & 0.000 \\
$\mathrm{D}_{5}$ & $1.43 \mathrm{E}-05$ & $1.36 \mathrm{E}-05$ & $1.36 \mathrm{E}-05$ & 4.56 & 0.000 \\
$\mathrm{D}_{6}$ & $1.09 \mathrm{E}-06$ & $1.04 \mathrm{E}-06$ & $1.04 \mathrm{E}-06$ & 4.35 & 0.000 \\
O-D4 & $8.63 \mathrm{E}-08$ & $5.89 \mathrm{E}-08$ & $5.89 \mathrm{E}-08$ & 31.73 & 0.027 \\
O-D5 & $2.85 \mathrm{E}-07$ & $2.03 \mathrm{E}-07$ & $2.03 \mathrm{E}-07$ & 28.71 & 0.019 \\
O-D6 & $2.58 \mathrm{E}-08$ & $1.90 \mathrm{E}-08$ & $1.90 \mathrm{E}-08$ & 26.43 & 0.014 \\
\hline
\end{tabular}




\section{Section S3: Gridded Population Data}

Gridded population was downloaded from EPA 2011 Version 6.0 Air Emissions Modeling Platform data https://www.epa.gov/air-emissions-modeling/2011-version-60-platform.

Specifically, we used population spatial surrogates. U.S. gridded population data is based on 2010 census data, Canada on 2001 census data, and Mexico from 1999/2000 census data. U.S. and Mexico population data was already gridded to the $36 \mathrm{~km}$ domain but Canada data was regridded from $12 \mathrm{~km}$. 


\section{Section S4: Cyclic Siloxane Emission Rates}

Table S2: Table of cVMS emission estimates. Numbers with superscripts correspond to ${ }^{\mathrm{a}}$ mean, ${ }^{\mathrm{b}}$ range, ${ }^{\mathrm{c}}$ median, and ${ }^{\mathrm{d}}$ maximum emission rates. Horii and Kannan (2008), Wang et al. (2009), and Dudzina et al. (2014) report exposure rate instead of air emissions. The values are likely to be similar to air emissions for Wang et al. (2009) since the products analyzed are expected to fully volatilize, the Horii and Kannan (2008) values are likely higher than air emissions since products washed down the drain are not distinguished, and the Dudzina et al. (2014) values are likely lower than air emissions since secondary volatilization from down the drain products is not included.

\begin{tabular}{|c|c|c|c|c|c|}
\hline \multirow[b]{2}{*}{ Location } & \multirow[b]{2}{*}{ Method } & \multicolumn{3}{|c|}{ Emission Rate (mg person ${ }^{-1}$ day $^{-1}$ ) } & \multirow[b]{2}{*}{ Reference } \\
\hline & & $\mathbf{D}_{4}$ & $\mathrm{D}_{5}$ & $\mathbf{D}_{6}$ & \\
\hline U.S. and Canada & $\begin{array}{l}\text { McLachlan et al. (2010) } D_{5} \text { estimate and } \\
\text { Chicago measurements }\end{array}$ & 32.8 & 135 & 6.10 & This work \\
\hline Mexico & $\begin{array}{l}\text { McLachlan et al. (2010) } D_{5} \text { estimate and } \\
\text { Chicago measurements }\end{array}$ & 5.92 & 24.4 & 1.10 & This work \\
\hline Berkley, CA, USA & Indoor classroom measurements & $(0.048-30.5)^{b}$ & $(4.39-235)^{b}$ & $(0.46-7.27)^{b}$ & Tang et al. (2015) \\
\hline Zurich, Switzerland & Model back-calculated & - & $310^{\mathrm{a}}(170-690)^{\mathrm{b}}$ & $36^{a}(19-81)^{b}$ & Buser et al. (2013) \\
\hline Chicago, IL, USA & Model back-calculated & - & $190^{\mathrm{a}}(100-420)^{\mathrm{b}}$ & - & Buser et al. (2014) \\
\hline U.S. & Dow Corning provided emissions & 90 & 137 & - & Navea et al. (2011) \\
\hline Iowa City, IA, USA & Indoor lab measurements & $(0.0090-0.027)^{b}$ & $(29-590)^{b}$ & - & Yucuis et al. (2013) \\
\hline Canada & $\begin{array}{l}\text { Personal care product } D_{5} \text { usage estimate } \\
(3,300 \mathrm{t} / \mathrm{yr})\end{array}$ & - & 260 & - & Buser et al. (2014) \\
\hline U.S. & $\begin{array}{l}\text { Exposure estimate from personal care } \\
\text { products }\end{array}$ & $1.08^{\mathrm{a}}$ & $233^{\mathrm{a}}$ & $22.2^{\mathrm{a}}$ & $\begin{array}{l}\text { Horii and Kannan } \\
\text { (2008) }\end{array}$ \\
\hline Canada & $\begin{array}{l}\text { Exposure estimate from lotion and } \\
\text { antiperspirant products }\end{array}$ & $98.6^{d}$ & $900^{d}$ & - & Wang et al. (2009) \\
\hline $\begin{array}{l}\text { Netherlands and } \\
\text { Switzerland }\end{array}$ & $\begin{array}{l}\text { Exposure estimate from personal care } \\
\text { products }\end{array}$ & $0.08^{c}\left(10.8^{d}\right)$ & $260^{c}\left(1,224^{d}\right)$ & - & Dudzina et al. (2014) \\
\hline Portugal & $\begin{array}{l}\text { Air emission estimate from personal care } \\
\text { products }\end{array}$ & $\begin{array}{c}0.130^{\mathrm{a}} \\
(0.00131-0.519)^{\mathrm{b}}\end{array}$ & $\begin{array}{c}0.815^{\mathrm{a}} \\
(0.00175-3.13)^{\mathrm{b}}\end{array}$ & $\begin{array}{c}0.500^{\mathrm{a}} \\
(0.00085-2.07)^{\mathrm{b}}\end{array}$ & Capela et al. (2016) \\
\hline
\end{tabular}




\section{Section S5: Calculation of $\mathrm{NO}_{\mathrm{x}} / \mathrm{NO}_{\mathrm{y}}$ Atmospheric Age}

$\mathrm{D}_{4}$ and $\mathrm{D}_{6}$ emission rates were estimated using Chicago outdoor concentrations from Yucuis et al. (2013) multiplied by the $\mathrm{D}_{5}$ emission rate. Chicago was assumed to be representative of fresh concentrations and hence the emission ratios. Due to different $\mathrm{OH}$ reactivity rates, product ratios change with photochemical age. $\mathrm{NO}_{\mathrm{x}}$ and $\mathrm{NO}_{\mathrm{y}}$ measurements from a Chicago EPA measurement site was used to estimate the photochemical age to verify the Chicago measurements represent fresh emissions. $\mathrm{NO}_{\mathrm{x}}$ and $\mathrm{NO}_{\mathrm{y}}$ has been used previously to estimate photochemical age assuming $\mathrm{NO}_{\mathrm{y}}$ is formed by $\mathrm{NO}_{2}+\mathrm{OH} \rightarrow \mathrm{HNO}_{3}$ (Slowik et al., 2011).

Hourly $\mathrm{NO}_{\mathrm{x}}$ and $\mathrm{NO}_{\mathrm{y}}$ data was extracted from the Northbrook, IL EPA monitoring site. Data was downloaded from Air Quality System Data Mart (http://www.epa.gov/ttn/airs/aqsdatamart). The analyzed period matched the Chicago cVMS measurements (August $13-21,2011$ ). Hourly $\mathrm{NO}_{\mathrm{x}} / \mathrm{NO}_{\mathrm{y}}$ ratios were calculated for hours that both $\mathrm{NO}_{\mathrm{x}}$ and $\mathrm{NO}_{\mathrm{y}}$ measurements were available, and then averaged for the measurement period. The cVMS concentrations were calculated by averaging the primary and duplicate Chicago measurements excluding Sample 6 due to being an outlier as discussed in Yucuis et al. (2013). Several $\mathrm{D}_{6}$ measurements were below the limit of quantification and were treated as $\mathrm{LOQ} / \sqrt{2}$.

The photochemical age was calculated using Equation $\mathrm{S} 1$. The $\mathrm{NO}_{\mathrm{x}} / \mathrm{NO}_{\mathrm{y}}$ ratio was 0.864 , OH concentration $1.33 \times 10^{6}$ molecules $\mathrm{cm}^{-3}$ interpolated for Chicago's latitude from Spivakovsky et al. (2000), and the $\mathrm{k}_{\mathrm{NO} 2}$ rate constant $1.08 \times 10^{-11} \mathrm{~cm}^{3} \mathrm{~mole}^{-1} \mathrm{~s}^{-1}$ estimated from the JPL 2011 parameterization for $295 \mathrm{~K}$ and surface pressure (Sander et al., 2011). The calculated age was determined to be 2.83 hours. Plugging in this age to Equation S2, the corrected emission ratio was calculated. $[\mathrm{A}]_{0} /[\mathrm{B}]_{0}$ represents the photochemically corrected emission ratio, $[\mathrm{A}] /[\mathrm{B}]$ the measured Chicago siloxane concentrations, $t$ the photochemical age, and $\mathrm{k}_{\mathrm{A}}$ and $\mathrm{k}_{\mathrm{B}}$ the respective siloxane $\mathrm{OH}$ rate constants. Corrected $\mathrm{D}_{4} / \mathrm{D}_{5}$ and $\mathrm{D}_{6} / \mathrm{D}_{5}$ emission ratios differed by less than $1 \%$ of the measured ratios. We therefore used the measured concentration ratios without photochemical age correction.

$$
\begin{gathered}
t=\frac{-\ln \left(\frac{[N O]_{x}}{[N O]_{y}}\right)}{[O H] k_{N O_{2}}} \\
\left(\frac{[A]_{0}}{[B]_{0}}\right)=\frac{\left(\frac{[A]}{[B]}\right)}{e^{-\left[t *[O H] *\left(k_{A}-k_{B}\right)\right]}}
\end{gathered}
$$




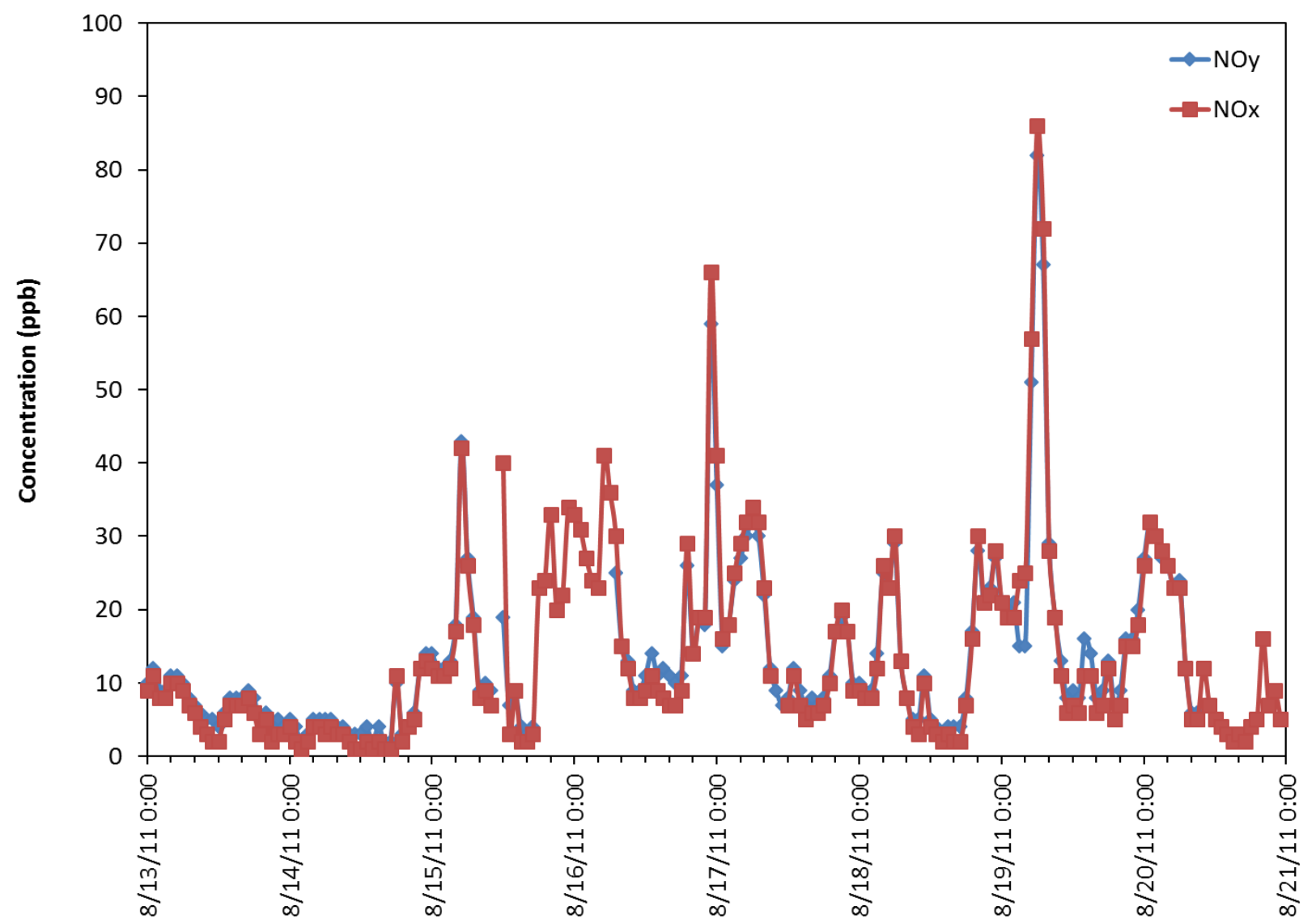

Figure S2: Northbrook hourly $\mathrm{NO}_{\mathrm{x}}$ and $\mathrm{NO}_{\mathrm{y}}$ measurements.

\section{Section S6: $D_{4}$ and $D_{6}$ Boundary Concentrations}

Cyclic siloxane boundary conditions were generated from the Danish Eulerian Hemispheric Model (DEHM) which simulated D5 at $150 \mathrm{~km}$ resolution for the Northern Hemisphere (Hansen et al., 2008; McLachlan et al., 2010). The DEHM model provided monthly averaged, horizontally and vertically resolved $\mathrm{D}_{5}$ concentrations. $\mathrm{D}_{4}$ and $\mathrm{D}_{6}$ boundary concentrations were estimated using the DEHM $\mathrm{D}_{5}$ concentrations multiplied by an $\mathrm{OH}$ dependent $\mathrm{D}_{4} / \mathrm{D}_{5}$ and $\mathrm{D}_{6} / \mathrm{D}_{5}$ ratio.

Since cVMS OH rate constants vary between species, an aged background ratio will differ from a fresh emission ratio and will also be dependent on the seasonally varying $\mathrm{OH}$ concentrations. $\mathrm{D}_{4} / \mathrm{D}_{5}$ and $\mathrm{D}_{6} / \mathrm{D}_{5}$ boundary ratios were calculated using spring cVMS atmospheric measurements at a rural site in Point Reyes, CA (Genualdi et al., 2011). Using Equation S3, combined with the aged Point Reyes measurements and the Chicago emission measurements, we were able to calculate a photochemical age. Here $t$ is the photochemical age, $[\mathrm{A}] /[\mathrm{B}]$ represents the measured siloxane ratio at Point Reyes, $[\mathrm{A}]_{0} /[\mathrm{B}]_{0}$ the fresh emission siloxane ratio, $[\mathrm{OH}]$ the average $\mathrm{OH}$ concentration during the 3 -month measurement period determined for the latitude of Point Reyes (Spivakovsky et al., 2000), and k the respective cVMS 
$\mathrm{OH}$ rate constants. Using $\mathrm{D}_{4}$ and $\mathrm{D}_{5}$, a photochemical age of 17.6 days was calculated and used for all calculations.

The aged, $\mathrm{OH}$ dependent background $\mathrm{D}_{4} / \mathrm{D}_{5}$ and $\mathrm{D}_{6} / \mathrm{D}_{5}$ ratios were calculated using Equation $\mathrm{S} 4$. Here $t$ is 17.6 days, $[\mathrm{OH}]$ the monthly dependent $\mathrm{OH}$ concentration as fit from Spivakovsky et al. (2000), $[\mathrm{A}]_{0} /[\mathrm{B}]_{0}$ the fresh emission siloxane ratio, and $[\mathrm{A}] /[\mathrm{B}]$ represents the seasonally corrected boundary siloxane ratio. $\mathrm{D}_{4}$ and $\mathrm{D}_{6}$ concentrations were estimated by combining the seasonally specific boundary ratios with the DEHM modeled $\mathrm{D}_{5}$. The same ratios were used for all four boundaries.

$$
t=-\ln \left[\frac{\left(\frac{[A]}{[B]}\right)}{\left(\frac{[A]_{0}}{[B]_{0}}\right)}\right] * \frac{1}{[O H] *\left(k_{A}-k_{B}\right)}
$$

$$
\frac{[A]}{[B]}=\frac{[A]_{0}}{[B]_{0}} e^{-[O H] * t *\left(k_{A}-k_{B}\right)}
$$

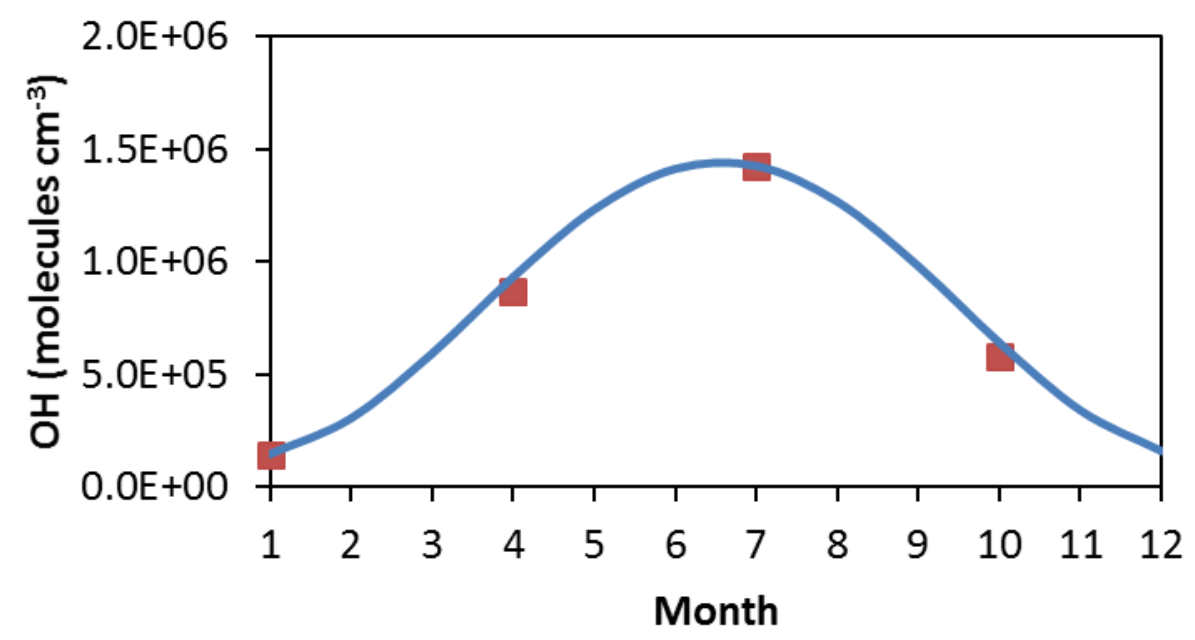

Figure S3: Spivakovsky et al. (2000) OH data for Point Reyes $\left(38^{\circ} \mathrm{N}\right)$ latitude. 

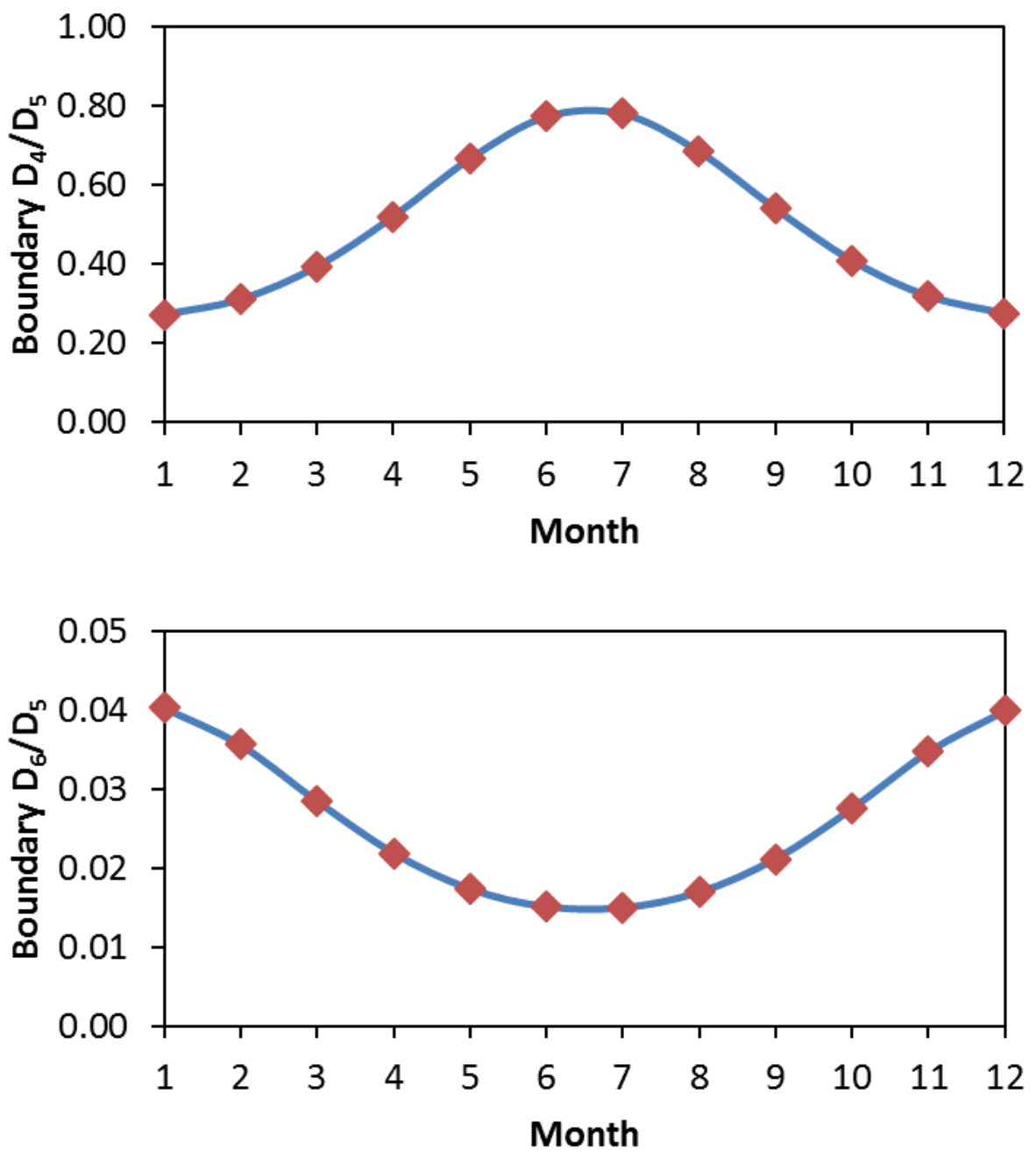

Figure S4: Monthly resolved $\mathrm{D}_{4} / \mathrm{D}_{5}$ and $\mathrm{D}_{6} / \mathrm{D}_{5}$ aged ratios. 


\section{Section S7: Analyzed Sites}

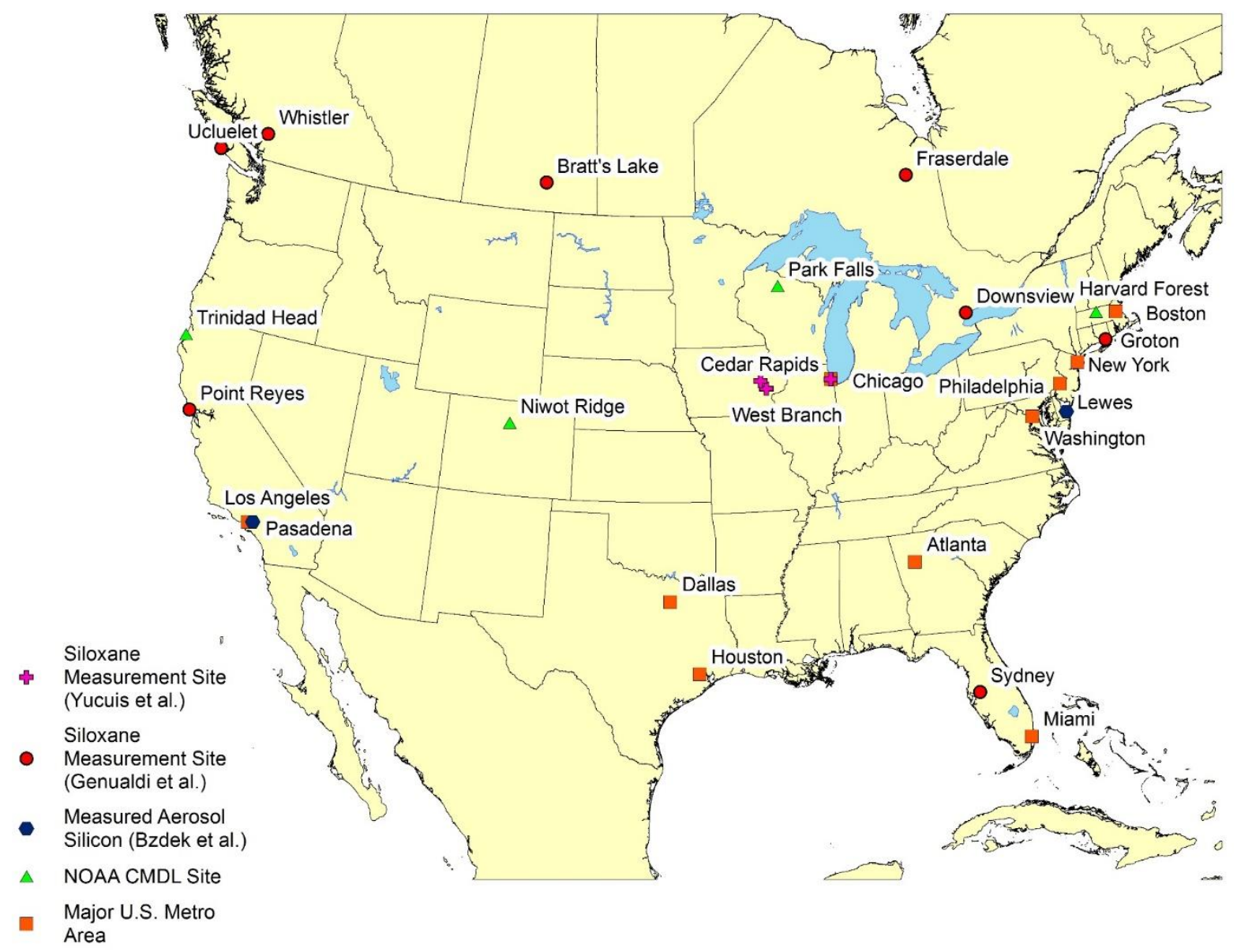

Figure S5: Analyzed 26 sites. 
Table S3: Classification of 26 sites. Sites with July $\mathrm{D}_{5}$ concentrations below $17 \mathrm{ng} \mathrm{m}^{-3}$ were classified as rural sites (in bold).

\begin{tabular}{|c|c|c|c|c|c|c|}
\hline \multirow[b]{2}{*}{ Location } & \multirow[b]{2}{*}{ Grid Cell Population } & \multirow[b]{2}{*}{ Classification } & \multicolumn{4}{|c|}{$D_{5}\left(n g m^{-3}\right)$} \\
\hline & & & January & April & July & October \\
\hline Fraserdale, ON, CAN & 12 & Rural & 2.88 & 1.93 & 0.76 & 5.88 \\
\hline Ucluelet, BC, CAN & 43 & Rural & 6.46 & 2.46 & 0.42 & 2.42 \\
\hline Niwot Ridge, CO, USA & 6,090 & Rural & 1.77 & 2.82 & 1.54 & 2.35 \\
\hline Park Falls, WI, USA & 7,265 & Rural & 5.91 & 3.59 & 3.12 & 8.31 \\
\hline Trinidad Head, CA, USA & 9,224 & Rural & 6.03 & 2.88 & 2.35 & 3.11 \\
\hline Whistler, BC, CAN & 9,588 & Rural & 5.40 & 4.47 & 2.73 & 4.21 \\
\hline West Branch, IA, USA & 20,291 & Rural & 13.24 & 8.88 & 8.82 & 18.30 \\
\hline Harvard Forest, MA, USA & 70,374 & Urban & 23.54 & 20.84 & 22.88 & 27.48 \\
\hline Lewes, DE, USA & 89,714 & Urban & 27.62 & 25.59 & 20.79 & 34.00 \\
\hline Groton, CT, USA & 100,246 & Urban & 29.99 & 43.90 & 44.27 & 30.80 \\
\hline Bratt's Lake, SK, CAN & 118,400 & Rural & 11.22 & 8.15 & 7.24 & 9.53 \\
\hline Point Reyes, CA, USA & 158,892 & Rural & 32.64 & 16.06 & 8.38 & 18.57 \\
\hline Cedar Rapids, IA, USA & 193,991 & Urban & 19.38 & 15.48 & 17.43 & 23.34 \\
\hline Sydney, FL, USA & 500,868 & Urban & 50.76 & 40.68 & 50.57 & 44.67 \\
\hline Dallas, TX, USA & $1,191,994$ & Urban & 83.52 & 63.43 & 52.06 & 93.46 \\
\hline Philadelphia, PA, USA & $1,286,968$ & Urban & 88.18 & 87.35 & 86.27 & 123.63 \\
\hline Atlanta, GA, USA & $1,413,880$ & Urban & 101.20 & 86.03 & 95.64 & 110.92 \\
\hline Downsview, ON, CAN & $1,481,245$ & Urban & 87.97 & 81.61 & 115.33 & 125.74 \\
\hline Boston, MA, USA & $1,506,543$ & Urban & 84.87 & 85.41 & 104.78 & 105.89 \\
\hline Miami, FL, USA & $1,550,514$ & Urban & 114.90 & 69.06 & 85.69 & 99.31 \\
\hline Washington, DC, USA & $1,719,747$ & Urban & 119.77 & 121.75 & 143.74 & 178.12 \\
\hline Houston, TX, USA & $1,806,399$ & Urban & 116.29 & 105.23 & 123.06 & 105.84 \\
\hline Pasadena, CA, USA & $1,979,007$ & Urban & 159.14 & 158.60 & 198.27 & 160.84 \\
\hline Chicago, IL, USA & $2,605,915$ & Urban & 139.34 & 131.79 & 167.80 & 164.02 \\
\hline Los Angeles, CA, USA & $4,133,658$ & Urban & 432.11 & 378.90 & 251.32 & 266.15 \\
\hline New York, NY, USA & $5,245,179$ & Urban & 234.37 & 227.78 & 265.35 & 301.30 \\
\hline
\end{tabular}


Table S4: Average monthly CMAQ modeled surface cVMS concentrations ( $\mathrm{ng} \mathrm{m}^{-3}$ ) sorted by population (highest at top of table) in analyzed grid cell. Minimum and maximum values in each column in boldface and italicized.

\begin{tabular}{|c|c|c|c|c|c|c|c|c|c|c|c|c|}
\hline \multirow[b]{2}{*}{ Site } & \multicolumn{4}{|c|}{$\mathrm{D}_{4}$} & \multicolumn{4}{|c|}{$\mathrm{D}_{5}$} & \multicolumn{4}{|c|}{$\mathrm{D}_{6}$} \\
\hline & January & April & July & October & January & April & July & October & January & April & July & October \\
\hline New York, NY, USA & 57.3 & 55.9 & 64.9 & 73.6 & 234 & 228 & 265 & 301 & 10.5 & 10.2 & 11.9 & 13.5 \\
\hline Los Angeles, CA, USA & 105 & 92.6 & 61.3 & 65.1 & 432 & 379 & 251 & 266 & 19.4 & 17.0 & 11.3 & 12.0 \\
\hline Chicago, IL, USA & 34.1 & 32.4 & 40.9 & 40.1 & 139 & 132 & 168 & 164 & 6.26 & 5.92 & 7.56 & 7.37 \\
\hline Pasadena, CA, USA & 38.9 & 39.0 & 48.6 & 39.5 & 159 & 159 & 198 & 161 & 7.15 & 7.11 & 8.90 & 7.21 \\
\hline Houston, TX, USA & 28.4 & 25.8 & 30.0 & 25.9 & 116 & 105 & 123 & 106 & 5.23 & 4.73 & 5.55 & 4.76 \\
\hline Washington, DC, USA & 29.4 & 30.0 & 35.1 & 43.5 & 120 & 122 & 144 & 178 & 5.38 & 5.46 & 6.47 & 8.01 \\
\hline Miami, FL, USA & 28.1 & 17.0 & 20.9 & 24.3 & 115 & 69.1 & 85.7 & 99.3 & 5.17 & 3.10 & 3.86 & 4.47 \\
\hline Boston, MA, USA & 21.0 & 21.3 & 25.9 & 26.1 & 84.9 & 85.4 & 105 & 106 & 3.79 & 3.81 & 4.69 & 4.74 \\
\hline Downsview, ON, CAN & 21.7 & 20.2 & 28.2 & 30.9 & 88.0 & 81.6 & 115 & 126 & 3.94 & 3.65 & 5.19 & 5.64 \\
\hline Atlanta, GA, USA & 24.8 & 21.2 & 23.4 & 27.1 & 101 & 86.0 & 95.6 & 111 & 4.54 & 3.86 & 4.30 & 4.98 \\
\hline Philadelphia, PA, USA & 21.7 & 21.7 & 21.3 & 30.4 & 88.2 & 87.3 & 86.3 & 124 & 3.95 & 3.90 & 3.86 & 5.54 \\
\hline Dallas, TX, USA & 20.5 & 15.6 & 12.8 & 22.9 & 83.5 & 63.4 & 52.1 & 93.5 & 3.75 & 2.84 & 2.34 & 4.20 \\
\hline Sydney, FL, USA & 12.5 & 10.2 & 12.6 & 11.0 & 50.8 & 40.7 & 50.6 & 44.7 & 2.27 & 1.81 & 2.25 & 2.00 \\
\hline Cedar Rapids, IA, USA & 4.91 & 4.06 & 4.37 & 5.88 & 19.4 & 15.5 & 17.4 & 23.3 & 0.853 & 0.675 & 0.777 & 1.03 \\
\hline Point Reyes, CA, USA & 8.04 & 4.12 & 2.10 & 4.63 & 32.6 & 16.1 & 8.38 & 18.6 & 1.46 & 0.707 & 0.373 & 0.826 \\
\hline Bratt's Lake, SK, CAN & 2.86 & 2.25 & 1.88 & 2.45 & 11.2 & 8.15 & 7.24 & 9.53 & 0.492 & 0.348 & 0.320 & 0.416 \\
\hline Groton, CT, USA & 7.62 & 11.2 & 11.3 & 7.91 & 30.0 & 43.9 & 44.3 & 30.8 & 1.32 & 1.93 & 1.95 & 1.34 \\
\hline Lewes, DE, USA & 6.99 & 6.67 & 5.31 & 8.61 & 27.6 & 25.6 & 20.8 & 34.0 & 1.22 & 1.12 & 0.915 & 1.50 \\
\hline Harvard Forest, MA, USA & 6.06 & 5.55 & 5.93 & 7.06 & 23.5 & 20.8 & 22.9 & 27.5 & 1.03 & 0.901 & 1.01 & 1.20 \\
\hline West Branch, IA, USA & 3.42 & 2.46 & 2.28 & 4.66 & 13.2 & 8.88 & 8.82 & 18.3 & 0.576 & 0.378 & 0.389 & 0.804 \\
\hline Whistler, BC, CAN & 1.39 & 1.30 & 0.728 & 1.11 & 5.40 & 4.47 & 2.73 & 4.21 & 0.235 & 0.185 & 0.118 & 0.181 \\
\hline Trinidad Head, CA, USA & 1.55 & 0.900 & 0.626 & 0.852 & 6.03 & 2.88 & 2.35 & 3.11 & 0.263 & 0.115 & 0.102 & 0.131 \\
\hline Park Falls, WI, USA & 1.67 & 1.19 & 0.911 & 2.23 & 5.91 & 3.59 & 3.12 & 8.31 & 0.242 & 0.138 & 0.131 & 0.354 \\
\hline Niwot Ridge, CO, USA & 0.478 & 0.829 & 0.468 & 0.649 & 1.77 & 2.82 & 1.54 & 2.35 & 0.0749 & 0.116 & 0.0623 & 0.0985 \\
\hline Ucluelet, BC, CAN & 1.66 & 0.827 & 0.142 & 0.687 & 6.46 & 2.46 & 0.423 & 2.42 & 0.282 & 0.0932 & 0.0170 & 0.0992 \\
\hline Fraserdale, ON, CAN & 1.06 & 0.869 & 0.350 & 1.71 & 2.88 & 1.93 & 0.756 & 5.88 & 0.0929 & 0.0559 & 0.0250 & 0.237 \\
\hline
\end{tabular}


Table S5: Average monthly CMAQ modeled surface oxidized cVMS concentrations ( $\mathrm{ng} \mathrm{m}^{-3}$ ) sorted by population (highest at top of table) in analyzed grid cell. Minimum and maximum values in each column in boldface and italicized.

\begin{tabular}{|c|c|c|c|c|c|c|c|c|c|c|c|c|}
\hline \multirow[b]{2}{*}{ Site } & \multicolumn{4}{|c|}{$O-D_{4}$} & \multicolumn{4}{|c|}{$O-D_{5}$} & \multicolumn{4}{|c|}{$0-D_{6}$} \\
\hline & January & April & July & October & January & April & July & October & January & April & July & October \\
\hline New York, NY, USA & 0.0760 & 0.383 & 0.782 & 0.404 & 0.454 & 2.11 & 4.60 & 2.46 & 0.0249 & 0.112 & 0.254 & 0.135 \\
\hline Los Angeles, CA, USA & 0.460 & 0.656 & 0.315 & 0.576 & 3.19 & 4.26 & 2.01 & 3.89 & 0.190 & 0.246 & 0.114 & 0.228 \\
\hline Chicago, IL, USA & 0.0622 & 0.278 & 0.427 & 0.219 & 0.359 & 1.32 & 2.30 & 1.27 & 0.0191 & 0.0642 & 0.122 & 0.0681 \\
\hline Pasadena, CA, USA & 0.389 & 0.655 & 0.720 & 0.666 & 2.49 & 3.92 & 4.46 & 4.21 & 0.142 & 0.215 & 0.248 & 0.237 \\
\hline Houston, TX, USA & 0.133 & 0.274 & 0.244 & 0.212 & 0.776 & 1.42 & 1.35 & 1.24 & 0.0417 & 0.0731 & 0.0726 & 0.0673 \\
\hline Washington, DC, USA & 0.0807 & 0.363 & 0.560 & 0.330 & 0.470 & 1.94 & 3.20 & 2.01 & 0.0253 & 0.101 & 0.175 & 0.112 \\
\hline Miami, FL, USA & 0.206 & 0.233 & 0.151 & 0.166 & 1.28 & 1.20 & 0.824 & 1.01 & 0.0711 & 0.0612 & 0.0439 & 0.0564 \\
\hline Boston, MA, USA & 0.0579 & 0.246 & 0.555 & 0.239 & 0.334 & 1.28 & 3.14 & 1.43 & 0.0180 & 0.0661 & 0.173 & 0.0779 \\
\hline Downsview, ON, CAN & 0.0424 & 0.210 & 0.373 & 0.162 & 0.246 & 0.994 & 2.08 & 0.948 & 0.0132 & 0.0483 & 0.113 & 0.0514 \\
\hline Atlanta, GA, USA & 0.176 & 0.402 & 0.464 & 0.344 & 1.06 & 1.99 & 2.52 & 2.03 & 0.0582 & 0.0989 & 0.134 & 0.110 \\
\hline Philadelphia, PA, USA & 0.0724 & 0.389 & 0.607 & 0.353 & 0.429 & 2.12 & 3.48 & 2.14 & 0.0234 & 0.112 & 0.191 & 0.118 \\
\hline Dallas, TX, USA & 0.179 & 0.286 & 0.299 & 0.221 & 1.04 & 1.39 & 1.54 & 1.26 & 0.0554 & 0.0687 & 0.0797 & 0.0666 \\
\hline Sydney, FL, USA & 0.186 & 0.340 & 0.528 & 0.234 & 1.14 & 1.78 & 3.01 & 1.40 & 0.0630 & 0.0914 & 0.163 & 0.0767 \\
\hline Cedar Rapids, IA, USA & 0.0531 & 0.233 & 0.264 & 0.154 & 0.295 & 1.00 & 1.29 & 0.864 & 0.0153 & 0.0454 & 0.0659 & 0.0455 \\
\hline Point Reyes, CA, USA & 0.0639 & 0.113 & 0.0639 & 0.0898 & 0.434 & 0.627 & 0.405 & 0.588 & 0.0256 & 0.0336 & 0.0237 & 0.0343 \\
\hline Bratt's Lake, SK, CAN & 0.00994 & 0.104 & 0.0971 & 0.0550 & 0.0547 & 0.362 & 0.394 & 0.282 & 0.00288 & 0.0134 & 0.0185 & 0.0138 \\
\hline Groton, CT, USA & 0.0691 & 0.254 & 0.644 & 0.247 & 0.427 & 1.56 & 4.27 & 1.60 & 0.0240 & 0.0900 & 0.257 & 0.0922 \\
\hline Lewes, DE, USA & 0.0759 & 0.340 & 0.478 & 0.291 & 0.449 & 1.85 & 2.69 & 1.78 & 0.0245 & 0.0989 & 0.147 & 0.0986 \\
\hline Harvard Forest, MA, USA & 0.0506 & 0.224 & 0.394 & 0.199 & 0.292 & 1.12 & 2.15 & 1.16 & 0.0159 & 0.0562 & 0.117 & 0.0626 \\
\hline West Branch, IA, USA & 0.0535 & 0.238 & 0.269 & 0.165 & 0.298 & 1.03 & 1.31 & 0.933 & 0.0155 & 0.0465 & 0.0669 & 0.0494 \\
\hline Whistler, BC, CAN & 0.0146 & 0.0863 & 0.0745 & 0.0306 & 0.0838 & 0.375 & 0.386 & 0.170 & 0.00453 & 0.0170 & 0.0201 & 0.00889 \\
\hline Trinidad Head, CA, USA & 0.0246 & 0.0742 & 0.0559 & 0.0516 & 0.164 & 0.345 & 0.340 & 0.329 & 0.00966 & 0.0163 & 0.0195 & 0.0189 \\
\hline Park Falls, WI, USA & 0.0213 & 0.125 & 0.172 & 0.104 & 0.114 & 0.486 & 0.778 & 0.582 & 0.00578 & 0.0200 & 0.0387 & 0.0306 \\
\hline Niwot Ridge, CO, USA & 0.0288 & 0.128 & 0.219 & 0.0994 & 0.156 & 0.569 & 1.00 & 0.549 & 0.00796 & 0.0265 & 0.0494 & 0.0285 \\
\hline Ucluelet, BC, CAN & 0.0138 & 0.0662 & 0.0142 & 0.0273 & 0.0955 & 0.288 & 0.0499 & 0.177 & 0.00587 & 0.0125 & 0.00210 & 0.0102 \\
\hline Fraserdale, ON, CAN & 0.0172 & 0.0557 & 0.0732 & 0.0668 & 0.0766 & 0.195 & 0.266 & 0.367 & 0.00328 & 0.00700 & 0.0119 & 0.0193 \\
\hline
\end{tabular}


Table S6: Monthly averaged modeled $\mathrm{OH}$ and meterology.

\begin{tabular}{|c|c|c|c|c|c|c|c|c|c|c|c|c|c|c|c|c|c|c|c|c|}
\hline \multirow[b]{2}{*}{ Site } & \multicolumn{4}{|c|}{$\mathrm{OH}\left(\right.$ molec $\left.\mathrm{cm}^{-3}\right)$} & \multicolumn{4}{|c|}{ Surface Temperature (K) } & \multicolumn{4}{|c|}{ Surface Pressure $(\mathrm{Pa})$} & \multicolumn{4}{|c|}{ Planetary Boundary Layer Height (m) } & \multicolumn{4}{|c|}{ Wind Speed $\left(\mathrm{m} \mathrm{s}^{-1}\right)$} \\
\hline & January & April & July & October & January & April & July & October & January & April & July & October & January & April & July & October & January & April & July & October \\
\hline New York, NY, USA & $2.15 E+05$ & $8.93 E+05$ & $2.12 E+06$ & $5.80 E+05$ & 269.3 & 284.2 & 296.4 & 286.5 & $1.01 E+05$ & $1.01 E+05$ & $1.01 E+05$ & $1.01 \mathrm{E}+05$ & 743.5 & 664.6 & 687.2 & 522.0 & 4.18 & 4.17 & 3.23 & 3.20 \\
\hline Los Angeles, CA, USA & $2.82 E+05$ & $5.38 \mathrm{E}+05$ & $6.00 E+05$ & $4.17 \mathrm{E}+05$ & 287.0 & 287.8 & 289.3 & 290.8 & $1.02 E+05$ & $1.01 E+05$ & $1.01 E+05$ & $1.01 E+05$ & 199.3 & 226.7 & 197.8 & 415.8 & 3.81 & 3.94 & 3.74 & 4.18 \\
\hline Chicago, IL, USA & $1.81 E+05$ & $1.07 E+06$ & $2.21 E+06$ & $5.13 E+05$ & 268.0 & 284.5 & 295.3 & 286.3 & $9.93 E+04$ & $9.90 E+04$ & $9.89 \mathrm{E}+04$ & $9.89 \mathrm{E}+04$ & 466.3 & 770.1 & 645.7 & 483.3 & 4.81 & 5.09 & 3.54 & 4.20 \\
\hline Pasadena, CA, USA & $5.35 E+05$ & $1.46 \mathrm{E}+06$ & $1.43 \mathrm{E}+06$ & $8.58 E+05$ & 287.1 & 290.2 & 292.6 & 291.1 & $9.98 \mathrm{E}+04$ & $9.94 \mathrm{E}+04$ & $9.93 E+04$ & $9.92 E+04$ & 417.2 & 614.6 & 376.5 & 494.8 & 3.14 & 3.17 & 2.77 & 2.91 \\
\hline Washington, DC, USA & $2.46 \mathrm{E}+05$ & $1.02 E+06$ & $2.10 \mathrm{E}+06$ & $6.47 E+05$ & 273.1 & 286.5 & 297.6 & 287.6 & $1.01 E+05$ & $1.01 E+05$ & $1.01 E+05$ & $1.01 E+05$ & 646.5 & 609.1 & 545.4 & 376.2 & 3.48 & 3.69 & 2.70 & 2.53 \\
\hline Miami, FL, USA & $1.03 E+06$ & $2.26 \mathrm{E}+06$ & $3.04 E+06$ & $1.49 \mathrm{E}+06$ & 292.4 & 295.6 & 300.5 & 298.4 & $1.02 E+05$ & $1.02 E+05$ & $1.01 E+05$ & $1.01 \mathrm{E}+05$ & 473.0 & 738.9 & 488.8 & 495.1 & 3.02 & 3.99 & 2.97 & 3.25 \\
\hline Boston, MA, USA & $2.29 \mathrm{E}+05$ & $7.48 \mathrm{E}+05$ & $1.96 \mathrm{E}+06$ & $5.60 \mathrm{E}+05$ & 266.4 & 281.8 & 294.6 & 284.7 & $1.01 E+05$ & $1.01 \mathrm{E}+05$ & $1.01 \mathrm{E}+05$ & $1.01 \mathrm{E}+05$ & 718.9 & 585.0 & 611.2 & 507.6 & 4.34 & 4.09 & 3.07 & 3.46 \\
\hline Downsview, ON, CAN & $2.13 E+05$ & $1.01 E+06$ & $1.95 E+06$ & $4.84 E+05$ & 264.2 & 279.9 & 292.7 & 283.7 & $9.81 E+04$ & $9.80 E+04$ & $9.80 \mathrm{E}+04$ & $9.82 E+04$ & 561.0 & 648.7 & 568.3 & 476.8 & 4.43 & 4.58 & 3.23 & 3.81 \\
\hline Atlanta, GA, USA & $3.75 E+05$ & $1.38 \mathrm{E}+06$ & $2.56 \mathrm{E}+06$ & $8.82 E+05$ & 278.9 & 289.5 & 298.9 & 292.5 & $9.84 E+04$ & $9.82 E+04$ & $9.81 E+04$ & $9.82 E+04$ & 351.3 & 714.6 & 618.2 & 450.2 & 3.75 & 3.65 & 2.96 & 2.94 \\
\hline Philadelphia, PA, USA & $2.31 E+05$ & $1.01 E+06$ & $2.20 E+06$ & $5.96 \mathrm{E}+05$ & 270.2 & 285.1 & 296.8 & 286.4 & $1.01 E+05$ & $1.01 E+05$ & $1.01 E+05$ & $1.01 E+05$ & 701.2 & 639.5 & 646.5 & 437.0 & 4.21 & 3.98 & 3.15 & 2.90 \\
\hline Sydney, FL, USA & $6.85 E+05$ & $1.99 E+06$ & $2.84 E+06$ & $1.41 E+06$ & 289.6 & 294.2 & 300.5 & 297.7 & $1.02 E+05$ & $1.01 E+05$ & $1.01 E+05$ & $1.01 E+05$ & 431.6 & 689.1 & 433.4 & 529.2 & 3.28 & 3.63 & 2.57 & 3.13 \\
\hline Cedar Rapids, IA, USA & $2.09 \mathrm{E}+05$ & $1.05 E+06$ & $2.22 E+06$ & $5.14 E+05$ & 267.0 & 285.4 & 295.2 & 285.9 & $9.88 \mathrm{E}+04$ & $9.84 E+04$ & $9.83 E+04$ & $9.83 E+04$ & 413.9 & 720.2 & 523.9 & 457.5 & 5.22 & 4.98 & 3.30 & 4.59 \\
\hline Point Reyes, CA, USA & $5.73 E+05$ & $1.79 E+06$ & $2.72 E+06$ & $1.19 \mathrm{E}+06$ & 284.2 & 284.9 & 286.3 & 287.6 & $1.02 \mathrm{E}+05$ & $1.01 E+05$ & $1.01 E+05$ & $1.01 \mathrm{E}+05$ & 382.7 & 201.4 & 93.7 & 242.5 & 6.11 & 5.65 & 5.18 & 5.55 \\
\hline Bratt's Lake, SK, CAN & $9.83 E+04$ & $1.51 E+06$ & $2.27 E+06$ & $5.37 E+05$ & 257.6 & 279.6 & 291.9 & 278.6 & $9.44 E+04$ & $9.42 E+04$ & $9.41 E+04$ & $9.39 \mathrm{E}+04$ & 244.2 & 849.3 & 665.2 & 420.6 & 4.11 & 4.99 & 3.88 & 4.49 \\
\hline Groton, CT, USA & $2.38 \mathrm{E}+05$ & $6.82 \mathrm{E}+05$ & $1.64 E+06$ & $5.87 E+05$ & 270.4 & 279.9 & 293.6 & 287.2 & $1.01 E+05$ & $1.01 \mathrm{E}+05$ & $1.01 E+05$ & $1.01 E+05$ & 1023.0 & 151.3 & 103.1 & 686.9 & 9.86 & 5.46 & 4.16 & 6.62 \\
\hline Lewes, DE, USA & $2.40 E+05$ & $9.40 E+05$ & $1.92 E+06$ & $6.48 E+05$ & 272.8 & 285.6 & 297.7 & 287.9 & $1.01 E+05$ & $1.01 E+05$ & $1.01 E+05$ & $1.01 E+05$ & 562.0 & 545.7 & 560.2 & 416.1 & 4.33 & 4.20 & 3.08 & 2.97 \\
\hline Harvard Forest, MA, USA & $2.03 E+05$ & $7.81 E+05$ & $1.86 \mathrm{E}+06$ & $5.17 E+05$ & 264.6 & 280.9 & 293.3 & 283.3 & $9.80 E+04$ & $9.81 \mathrm{E}+04$ & $9.83 E+04$ & $9.85 E+04$ & 679.8 & 635.1 & 578.9 & 511.1 & 4.64 & 4.14 & 2.88 & 3.28 \\
\hline West Branch, IA, USA & $2.14 E+05$ & $1.07 E+06$ & $2.20 \mathrm{E}+06$ & $5.33 E+05$ & 267.8 & 285.9 & 295.7 & 286.4 & $9.92 E+04$ & $9.88 \mathrm{E}+04$ & $9.87 E+04$ & $9.87 E+04$ & 420.6 & 748.2 & 531.5 & 455.7 & 5.21 & 5.08 & 3.30 & 4.64 \\
\hline Whistler, BC, CAN & $2.01 E+05$ & $8.29 E+05$ & $8.97 E+05$ & $4.14 E+05$ & 270.7 & 278.3 & 287.5 & 278.6 & $8.84 \mathrm{E}+04$ & $8.88 \mathrm{E}+04$ & $8.90 \mathrm{E}+04$ & $8.84 E+04$ & 237.6 & 590.0 & 650.2 & 343.1 & 3.79 & 3.20 & 3.24 & 3.78 \\
\hline Trinidad Head, CA, USA & $3.63 E+05$ & $1.31 \mathrm{E}+06$ & $2.30 \mathrm{E}+06$ & $6.99 \mathrm{E}+05$ & 283.9 & 284.9 & 288.4 & 287.8 & $1.02 \mathrm{E}+05$ & $1.02 \mathrm{E}+05$ & $1.01 \mathrm{E}+05$ & $1.01 \mathrm{E}+05$ & 404.9 & 259.7 & 144.2 & 285.6 & 6.21 & 6.14 & 6.24 & 5.25 \\
\hline Park Falls, WI, USA & $1.61 E+05$ & $7.54 E+05$ & $1.97 E+06$ & $4.36 \mathrm{E}+05$ & 260.7 & 278.3 & 291.3 & 281.7 & $9.61 \mathrm{E}+04$ & $9.59 \mathrm{E}+04$ & $9.60 E+04$ & $9.58 \mathrm{E}+04$ & 434.1 & 509.3 & 571.7 & 450.4 & 4.54 & 4.08 & 3.43 & 4.53 \\
\hline Niwot Ridge, CO, USA & $4.90 \mathrm{E}+05$ & $1.51 \mathrm{E}+06$ & $3.26 \mathrm{E}+06$ & $9.85 E+05$ & 264.1 & 273.0 & 285.2 & 275.3 & $7.03 E+04$ & $7.04 \mathrm{E}+04$ & $7.13 \mathrm{E}+04$ & $7.06 \mathrm{E}+04$ & 389.3 & 747.9 & 999.6 & 700.4 & 4.76 & 5.02 & 3.58 & 4.97 \\
\hline Ucluelet, BC, CAN & $1.53 E+05$ & $9.79 E+05$ & $1.15 E+06$ & $4.27 E+05$ & 280.2 & 282.7 & 288.3 & 285.8 & $1.01 E+05$ & $1.02 E+05$ & $1.01 E+05$ & $1.01 E+05$ & 536.8 & 275.8 & 200.3 & 425.1 & 8.89 & 5.08 & 4.21 & 5.98 \\
\hline
\end{tabular}




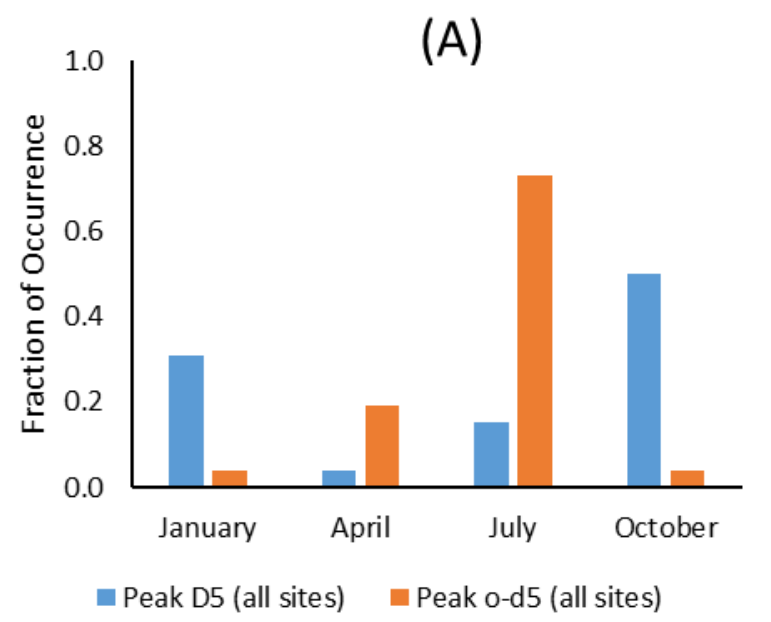

(D)

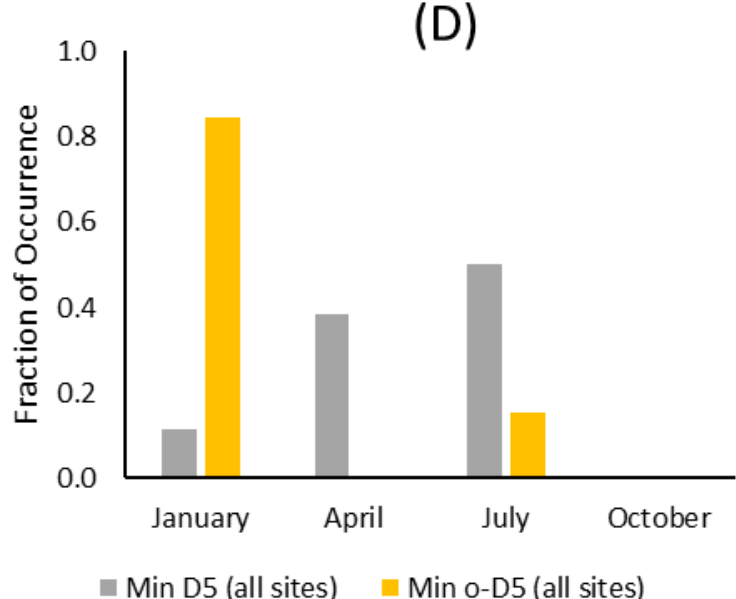

Min D5 (all sites) Min o-D5 (all sites)
(B)

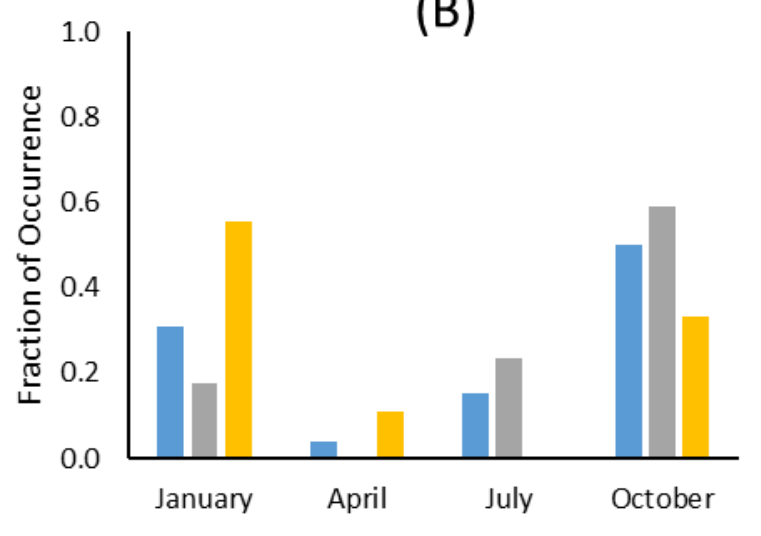

@ Peak D5 (all sites) $₫$ Peak D5 (urban) $₫$ Peak D5 (rural)

(E)

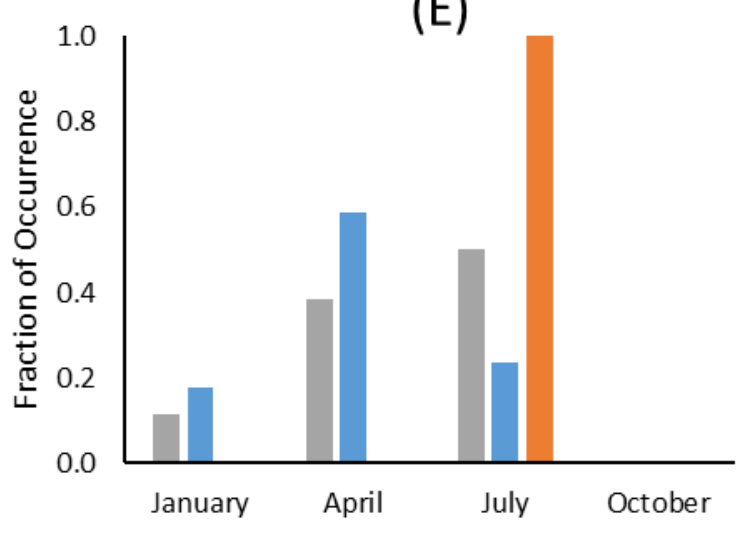

min D5 (all sites) $\square$ Min D5 (urban) $\square$ Min D5 (rural)
(C)

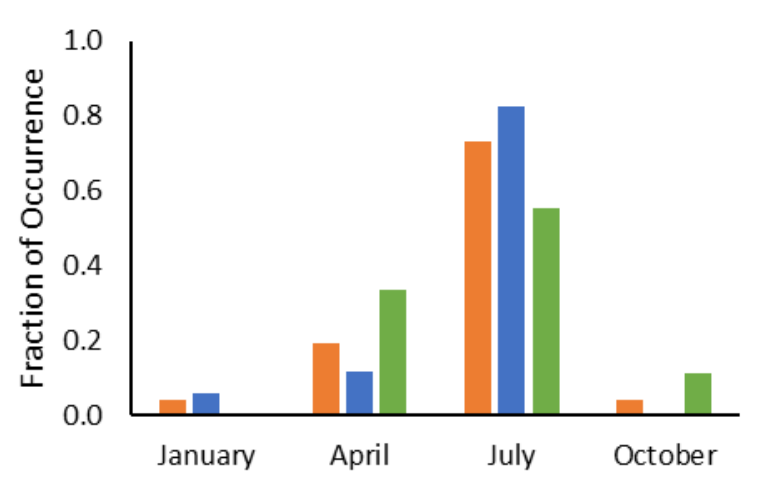

— Peak o-d5 (all sites) — Peak o-D5 (urban)

- Peak o-D5 (rural)

(F)

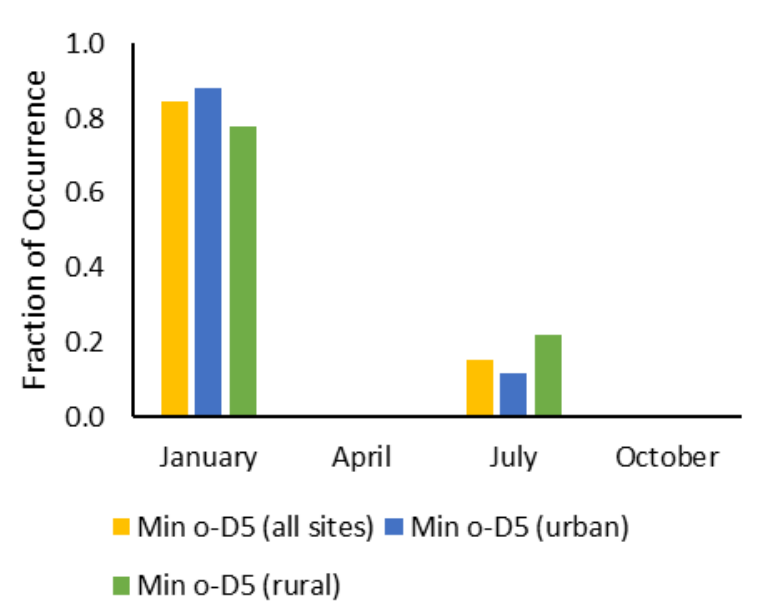

Figure S6: Seasonal trends of $\mathrm{D}_{5}$ and o- $\mathrm{D}_{5}$ concentrations for the 26 analyzed sites. Of the four months modeled, the month of highest or lowest average $\mathrm{D}_{5}$ and $\mathrm{o}-\mathrm{D}_{5}$ concentrations are tabulated. Panel (A) shows monthly occurrence of highest $\mathrm{D}_{5}$ and o- $\mathrm{D}_{5}$ concentrations for all sites, (B) occurrence of highest $\mathrm{D}_{5}$ for urban and rural sites, (C) occurrence of highest o- $\mathrm{D}_{5}$ for urban and rural sites, (D) occurrence of lowest $\mathrm{D}_{5}$ and o- $\mathrm{D}_{5}$ for all sites, (E) occurrence of lowest $\mathrm{D}_{5}$ for urban and rural sites, and (F) occurrence of lowest o- $\mathrm{D}_{5}$ for urban and rural sites. 
Table S7: Monthly averaged modeled compound ratios.

\begin{tabular}{|c|c|c|c|c|c|c|c|c|c|c|c|c|}
\hline \multirow[b]{2}{*}{ Site } & \multicolumn{4}{|c|}{$D_{5} / D_{4}$} & \multicolumn{4}{|c|}{$D_{6} / D_{5}$} & \multicolumn{4}{|c|}{$\mathrm{SO}_{2} / \mathrm{cVMS}$} \\
\hline & January & April & July & October & January & April & July & October & January & April & July & October \\
\hline New York, NY, USA & 3.27 & 3.26 & 3.27 & 3.27 & 0.0375 & 0.0374 & 0.0375 & 0.0375 & 9,294 & 6,937 & 5,960 & 8,247 \\
\hline Los Angeles, CA, USA & 3.28 & 3.27 & 3.28 & 3.27 & 0.0375 & 0.0375 & 0.0375 & 0.0374 & 337 & 251 & 185 & 319 \\
\hline Chicago, IL, USA & 3.27 & 3.26 & 3.28 & 3.27 & 0.0375 & 0.0374 & 0.0376 & 0.0375 & 11,538 & 8,616 & 5,662 & 9,615 \\
\hline Pasadena, CA, USA & 3.27 & 3.26 & 3.26 & 3.26 & 0.0374 & 0.0374 & 0.0374 & 0.0374 & 761 & 283 & 266 & 503 \\
\hline Houston, TX, USA & 3.27 & 3.27 & 3.28 & 3.27 & 0.0375 & 0.0375 & 0.0376 & 0.0375 & 9,611 & 4,697 & 4,104 & 5,211 \\
\hline Washington, DC, USA & 3.26 & 3.25 & 3.27 & 3.27 & 0.0374 & 0.0374 & 0.0375 & 0.0375 & 33,544 & 24,260 & 21,360 & 24,316 \\
\hline Miami, FL, USA & 3.28 & 3.25 & 3.28 & 3.27 & 0.0375 & 0.0374 & 0.0375 & 0.0375 & 5,194 & 4,778 & 1,945 & 2,641 \\
\hline Boston, MA, USA & 3.24 & 3.21 & 3.23 & 3.24 & 0.0372 & 0.0372 & 0.0373 & 0.0373 & 15,531 & 12,303 & 9,211 & 14,497 \\
\hline Downsview, ON, CAN & 3.24 & 3.23 & 3.27 & 3.26 & 0.0373 & 0.0373 & 0.0375 & 0.0374 & 1,546 & 2,973 & 588 & 2,185 \\
\hline Atlanta, GA, USA & 3.26 & 3.24 & 3.27 & 3.27 & 0.0374 & 0.0374 & 0.0375 & 0.0375 & 30,866 & 28,251 & 18,309 & 22,427 \\
\hline Philadelphia, PA, USA & 3.25 & 3.22 & 3.24 & 3.25 & 0.0373 & 0.0372 & 0.0373 & 0.0373 & 20,139 & 17,350 & 15,060 & 17,912 \\
\hline Sydney, FL, USA & 3.24 & 3.20 & 3.22 & 3.24 & 0.0373 & 0.0371 & 0.0372 & 0.0373 & 21,592 & 16,777 & 7,641 & 12,545 \\
\hline Cedar Rapids, IA, USA & 3.16 & 3.05 & 3.19 & 3.18 & 0.0367 & 0.0363 & 0.0372 & 0.0368 & 144,645 & 126,766 & 97,014 & 89,098 \\
\hline Point Reyes, CA, USA & 3.25 & 3.12 & 3.19 & 3.21 & 0.0373 & 0.0367 & 0.0371 & 0.0371 & 2,581 & 2,732 & 2,436 & 5,866 \\
\hline Bratt's Lake, SK, CAN & 3.14 & 2.90 & 3.09 & 3.11 & 0.0366 & 0.0355 & 0.0368 & 0.0364 & 8,505 & 6,449 & 5,263 & 19,517 \\
\hline Groton, $\mathrm{CT}$, USA & 3.15 & 3.13 & 3.13 & 3.11 & 0.0366 & 0.0367 & 0.0367 & 0.0364 & 23,025 & 14,862 & 18,158 & 24,402 \\
\hline Lewes, DE, USA & 3.16 & 3.07 & 3.13 & 3.16 & 0.0367 & 0.0364 & 0.0367 & 0.0367 & 49,557 & 39,235 & 40,384 & 34,715 \\
\hline Harvard Forest, MA, USA & 3.11 & 3.01 & 3.09 & 3.12 & 0.0364 & 0.0360 & 0.0366 & 0.0364 & 25,561 & 30,775 & 20,004 & 32,611 \\
\hline West Branch, IA, USA & 3.10 & 2.89 & 3.10 & 3.14 & 0.0363 & 0.0354 & 0.0367 & 0.0366 & 207,140 & 217,583 & 194,708 & 115,970 \\
\hline Whistler, BC, CAN & 3.10 & 2.76 & 3.00 & 3.03 & 0.0363 & 0.0345 & 0.0361 & 0.0359 & 1,885 & 3,819 & 8,098 & 3,585 \\
\hline Trinidad Head, CA, USA & 3.10 & 2.56 & 3.00 & 2.92 & 0.0363 & 0.0332 & 0.0361 & 0.0351 & 7,626 & 22,248 & 33,099 & 30,096 \\
\hline Park Falls, WI, USA & 2.83 & 2.40 & 2.74 & 2.99 & 0.0341 & 0.0320 & 0.0350 & 0.0355 & 121,956 & 255,080 & 184,828 & 128,076 \\
\hline Niwot Ridge, CO, USA & 2.96 & 2.72 & 2.62 & 2.90 & 0.0353 & 0.0343 & 0.0338 & 0.0349 & 256,457 & 84,118 & 161,838 & 123,540 \\
\hline Ucluelet, BC, CAN & 3.11 & 2.38 & 2.38 & 2.82 & 0.0364 & 0.0316 & 0.0336 & 0.0341 & 3,929 & 3,915 & 2,625 & 7,795 \\
\hline
\end{tabular}




\section{Section S8: Linear Regression}

Table S8: Linear least-squares regression results of normalized $\mathrm{D}_{5}$ as a function of the inverse of normalized $\mathrm{OH}, \mathrm{PBL}$, and wind speed for the 26 sites. All values are dimensionless.

Normalization is through division by the July value of the variable at that location.

\begin{tabular}{|c|c|c|}
\hline \multicolumn{3}{|c|}{ All 26 Sites } \\
\hline Sites & Variable (Coefficient); variables with $p<0.1$ bolded & Adjusted $\mathrm{R}^{2}$ \\
\hline Urban & $\mathrm{OH}^{-1}(-0.0039)$ & -0.019 \\
\hline Urban & $\mathrm{PBL}^{-1}(0.48)$ & 0.333 \\
\hline Urban & WS $^{-1}(1.35)$ & 0.450 \\
\hline Urban & $\mathrm{PBL}^{-1} \cdot \mathrm{WS}^{-1}(0.52)$ & 0.492 \\
\hline Urban & $\mathrm{OH}^{-1}(0.0068), \mathrm{PBL}^{-1}(0.20), \mathbf{W S}^{-1}(1.11)$ & 0.489 \\
\hline Urban & $\mathrm{OH}^{-1}(0.0046), \mathrm{PBL}^{-1}(-0.32), \mathrm{WS}^{-1}(0.37), \mathrm{PBL}^{-1} \cdot \mathrm{WS}^{-1}(\mathbf{0 . 6 8})$ & 0.509 \\
\hline Urban & $\mathrm{OH}^{-1}(-0.0083), \mathrm{PBL}^{-1} \cdot \mathrm{WS}^{-1}(\mathbf{0 . 5 2})$ & 0.490 \\
\hline Urban & $\mathrm{PBL}^{-1}(-0.31), \mathrm{WS}^{-1}(0.29), \mathrm{PBL}^{-1} \cdot \mathrm{WS}^{-1}(0.70)$ & 0.517 \\
\hline Urban & $\mathrm{PBL}^{-1}(0.24), \mathrm{WS}^{-1}(1.01)$ & 0.496 \\
\hline Urban & $\mathrm{OH}^{-1}(-0.018), \mathrm{PBL}^{-1}(\mathbf{0 . 5 2})$ & 0.356 \\
\hline Urban & $\mathrm{OH}^{-1}(0.017), \mathrm{WS}^{-1}(1.46)$ & 0.471 \\
\hline Rural & $\mathrm{OH}^{-1}(0.0033)$ & -0.040 \\
\hline Rural & $\mathrm{PBL}^{-1}(-1.15)$ & 0.039 \\
\hline Rural & WS $^{-1}(-7.27)$ & 0.104 \\
\hline Rural & $\mathrm{PBL}^{-1} \cdot \mathrm{WS}^{-1}(-1.27)$ & 0.035 \\
\hline Rural & $\mathrm{OH}^{-1}(0.062), \mathrm{PBL}^{-1}(-1.32)$, WS $^{-1}(-7.00)$ & 0.119 \\
\hline Rural & $\mathrm{OH}^{-1}(-0.022), \mathrm{PBL}^{-1}(-18.02), \mathrm{WS}^{-1}(-23.79), \mathrm{PBL}^{-1} \cdot \mathrm{WS}^{-1}(19.69)$ & 0.425 \\
\hline Rural & $\mathrm{OH}^{-1}(0.093) \mathrm{PBL}^{-1} \cdot \mathrm{WS}^{-1}(-1.55)$ & 0.014 \\
\hline Rural & $\mathrm{PBL}^{-1}(-17.89), \mathrm{WS}^{-1}(-23.52), \mathrm{PBL}^{-1} \cdot \mathrm{WS}^{-1}(19.47)$ & 0.449 \\
\hline Rural & $\mathrm{PBL}^{-1}(-1.14), \mathrm{WS}^{-1}(-7.24)$ & 0.148 \\
\hline Rural & $\mathrm{OH}^{-1}(0.10), \mathrm{PBL}^{-1}(-1.43)$ & 0.020 \\
\hline Rural & $\mathrm{OH}^{-1}(-0.028), \mathrm{WS}^{-1}(-7.38)$ & 0.069 \\
\hline
\end{tabular}


Table S9: Linear least-squares regression results of normalized $\mathrm{D}_{5}$ as a function of the inverse of normalized OH, PBL, and wind speed excluding Canadian and Point Reyes sites. All values are dimensionless. Normalization is through division by the July value of the variable at that location.

\begin{tabular}{|c|c|c|}
\hline \multicolumn{3}{|c|}{ Without Canadian and Point Reyes Sites } \\
\hline Sites & Variable (Coefficient); variables with $p<0.1$ bolded & Adjusted $\mathrm{R}^{2}$ \\
\hline Urban & $\mathrm{OH}^{-1}(-0.0023)$ & -0.021 \\
\hline Urban & PBL $^{-1}(0.48)$ & 0.342 \\
\hline Urban & WS $^{-1}(1.31)$ & 0.438 \\
\hline Urban & $\mathrm{PBL}^{-1} \cdot \mathrm{WS}^{-1}(0.51)$ & 0.493 \\
\hline Urban & $\mathrm{OH}^{-1}(0.0081), \mathrm{PBL}^{-1}(0.20), \mathbf{W S}^{-1}(1.07)$ & 0.484 \\
\hline Urban & $\mathrm{OH}^{-1}(0.0057), \mathrm{PBL}^{-1}(-0.29), \mathrm{WS}^{-1}(0.38), \mathrm{PBL}^{-1} \cdot \mathrm{WS}^{-1}(0.64)$ & 0.501 \\
\hline Urban & $\mathrm{OH}^{-1}(-0.0070) \mathrm{PBL}^{-1} \cdot \mathbf{W S}^{-1}(\mathbf{0 . 5 1})$ & 0.487 \\
\hline Urban & $\mathrm{PBL}^{-1}(-0.28), \mathrm{WS}^{-1}(0.27), \mathrm{PBL}^{-1} \cdot \mathrm{WS}^{-1}(0.66)$ & 0.510 \\
\hline Urban & $\mathrm{PBL}^{-1}(0.25), \mathrm{WS}^{-1}(0.96)$ & 0.491 \\
\hline Urban & $\mathrm{OH}^{-1}(-0.017), \mathrm{PBL}^{-1}(\mathbf{0 . 5 1})$ & 0.359 \\
\hline Urban & $\mathrm{OH}^{-1}(0.019), \mathrm{WS}^{-1}(1.44)$ & 0.465 \\
\hline Rural & $\mathrm{OH}^{-1}(0.042)$ & -0.029 \\
\hline Rural & $\mathrm{PBL}^{-1}(-0.14)$ & -0.076 \\
\hline Rural & $W^{-1}(-0.0088)$ & -0.100 \\
\hline Rural & $\mathrm{PBL}^{-1} \cdot \mathrm{WS}^{-1}(-0.25)$ & -0.064 \\
\hline Rural & $\mathrm{OH}^{-1}(0.053), \mathrm{PBL}^{-1}(-0.28), \mathrm{WS}^{-1}(-0.29)$ & -0.204 \\
\hline Rural & $\mathrm{OH}^{-1}(0.047), \mathrm{PBL}^{-1}(0.94), \mathrm{WS}^{-1}(0.67), \mathrm{PBL}^{-1} \cdot \mathrm{WS}^{-1}(-1.56)$ & -0.366 \\
\hline Rural & $\mathrm{OH}^{-1}(0.052) \mathrm{PBL}^{-1} \cdot \mathrm{WS}^{-1}(-0.35)$ & -0.068 \\
\hline Rural & $\mathrm{PBL}^{-1}(2.37), \mathrm{WS}^{-1}(1.66), \mathrm{PBL}^{-1} \cdot \mathrm{WS}^{-1}(-3.34)$ & -0.282 \\
\hline Rural & $\mathrm{PBL}^{-1}(-0.21), \mathrm{WS}^{-1}(-0.41)$ & -0.183 \\
\hline Rural & $\mathrm{OH}^{-1}(0.054), \mathrm{PBL}^{-1}(-0.23)$ & -0.077 \\
\hline Rural & $\mathrm{OH}^{-1}(0.044), \mathrm{WS}^{-1}(0.21)$ & -0.139 \\
\hline
\end{tabular}



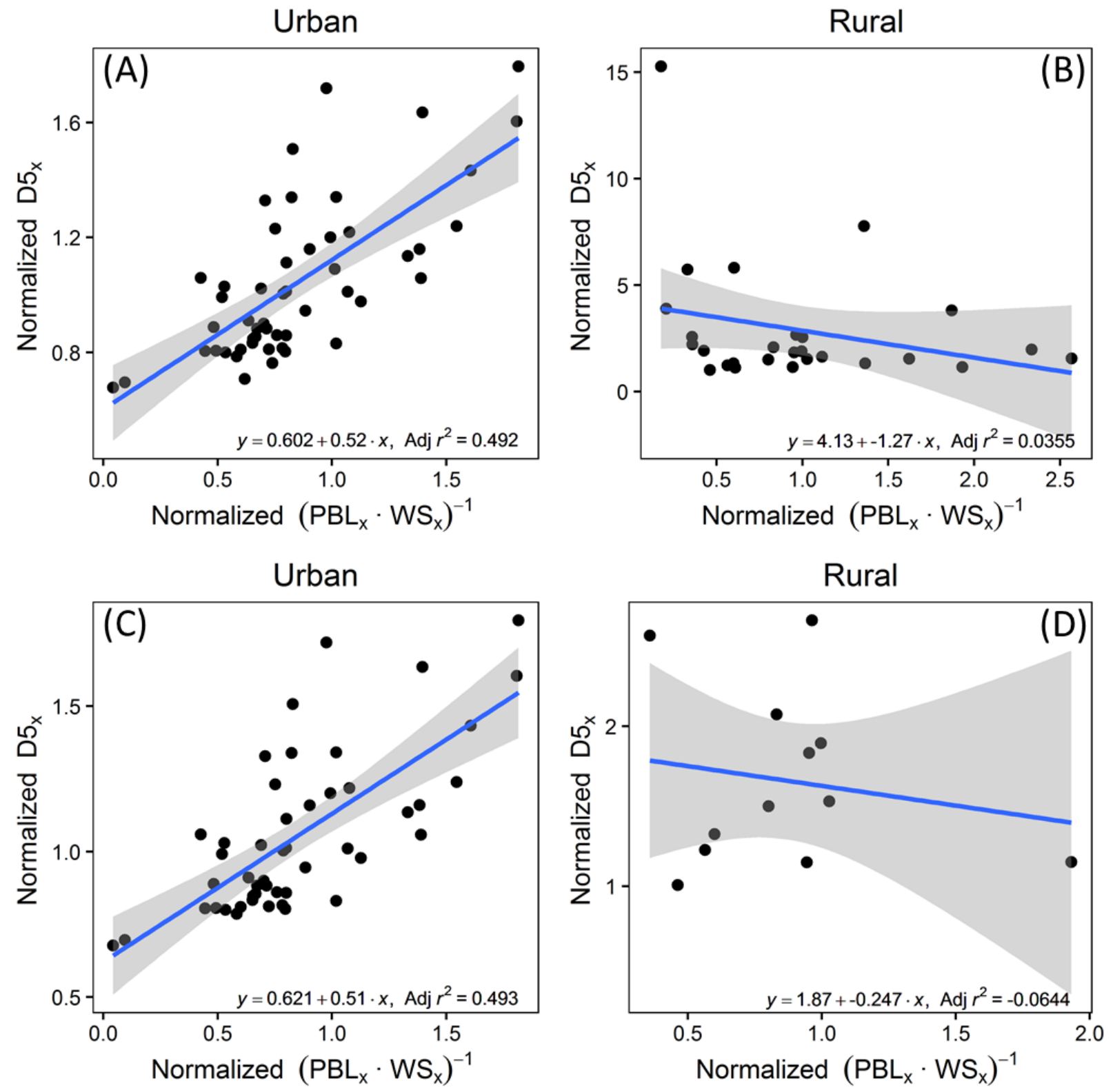

Figure S7: Linear least-squares regression fit of monthly averaged normalized $\mathrm{D}_{5}$ concentrations versus the inverse of normalized boundary layer height and normalized wind speed for all 26 analyzed sites urban (A) and rural (B) sites. The same sites excluding Canadian and Point Reyes, CA locations are also shown for urban (C) and rural (D) sites. Normalization is through division by the July value of the quantity. 

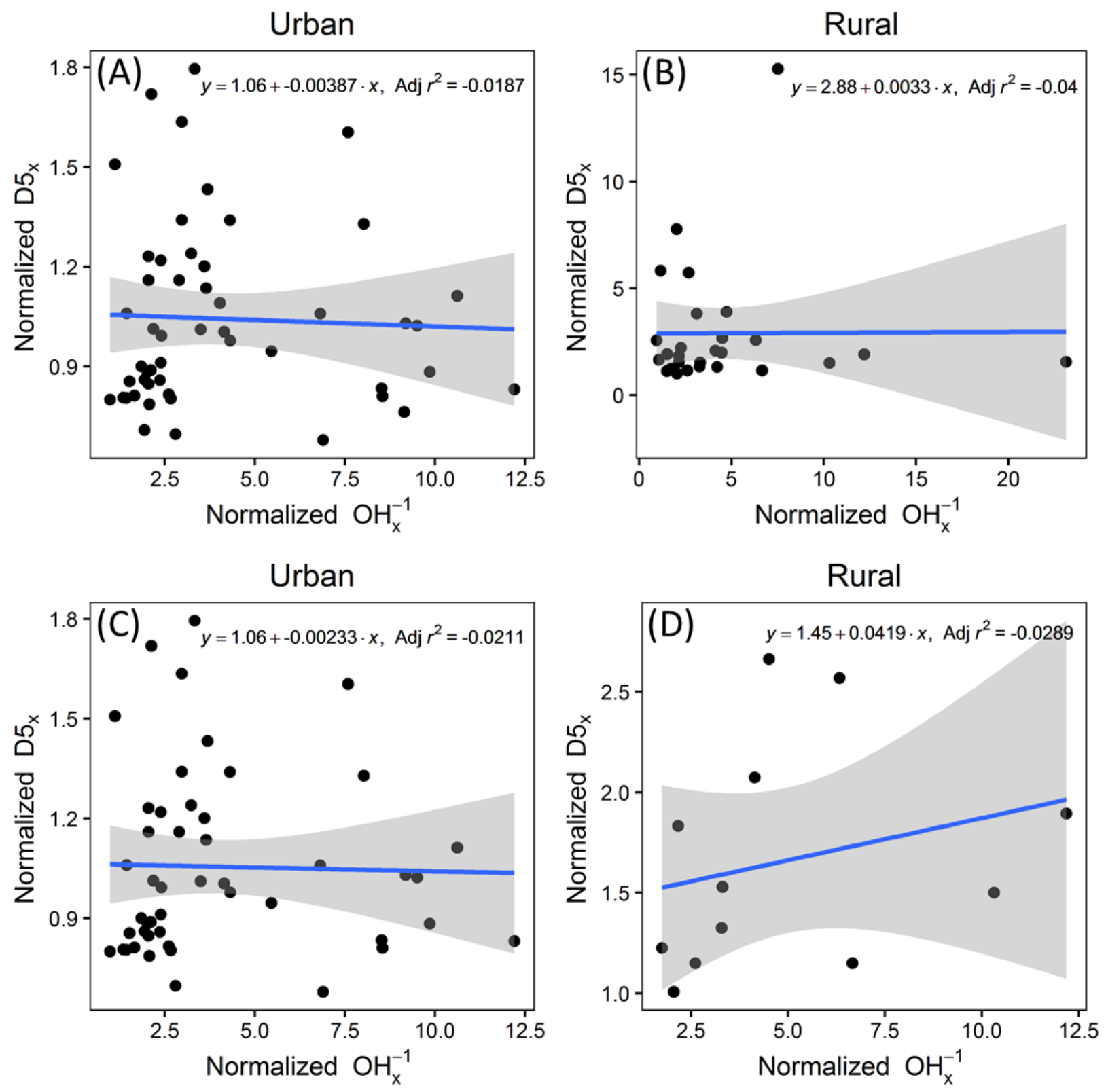

Figure S8: Linear least-squares regression fit of monthly averaged normalized $\mathrm{D}_{5}$ concentrations versus the inverse of normalized $\mathrm{OH}$ concentration for all 26 analyzed sites urban (A) and rural (B) sites. The same sites excluding Canadian and Point Reyes, CA locations are also shown for urban (C) and rural (D) sites. Normalization is through division by the July value of the quantity. 


\section{Section S9: Midwest Model Performance}

Table S10: Model performance to Yucuis et al. (2013) Midwest sites. Fractional bias can range from -2 to +2 while fractional error ranges from 0 to +2 .

\begin{tabular}{|c|c|c|c|c|c|c|c|c|c|}
\hline & \multicolumn{3}{|c|}{ Chicago } & \multicolumn{3}{|c|}{ Cedar Rapids } & \multicolumn{3}{|c|}{ West Branch } \\
\hline & $\mathbf{D}_{4}$ & $D_{5}$ & $D_{6}$ & $\mathbf{D}_{4}$ & $D_{5}$ & $D_{6}$ & $\mathrm{D}_{4}$ & $D_{5}$ & $D_{6}$ \\
\hline CMAQ Mean (ng m³) & 41.2 & 169 & 7.61 & 4.36 & 17.4 & 0.777 & 2.28 & 8.83 & 0.389 \\
\hline Yucuis et al. Mean (ng m $\mathrm{m}^{-3}$ ) & 56.3 & 232 & 10.1 & 18.9 & 47.9 & 5.56 & 9.94 & 20.1 & 1.65 \\
\hline Measured to Model Factor & 1.37 & 1.37 & 1.33 & 4.33 & 2.75 & 7.16 & 4.36 & 2.27 & 4.23 \\
\hline Frac. Bias & -0.309 & -0.313 & -0.281 & -1.25 & -0.933 & -1.51 & -1.25 & -0.777 & -1.23 \\
\hline Frac. Error & 0.309 & 0.313 & 0.281 & 1.25 & 0.933 & 1.51 & 1.25 & 0.777 & 1.23 \\
\hline Error & -15.1 & -62.7 & -2.49 & -14.5 & -30.5 & -4.79 & -7.66 & -11.2 & -1.26 \\
\hline \% Relative Error & -26.8 & -27.1 & -24.6 & -76.9 & -63.6 & -86.0 & -77.1 & -56.0 & -76.4 \\
\hline
\end{tabular}

Frac. Bias $=\left(\frac{m-o}{\frac{m+o}{2}}\right)$

Frac. Error $=\left(\frac{|m-o|}{\frac{m+o}{2}}\right)$

Error $=m-o$

$\%$ Relative Error $=\left(\frac{m-o}{o}\right) \times 100$ 


\section{Section S10: GAPS Model Performance}

Table S11: Measurement and model fractional bias, fractional error, and absolute error. Measurement concentrations are from Genualdi et al. (2011). Fractional bias can range from -2 to +2 while fractional error can range from 0 to +2 .

\begin{tabular}{|c|c|c|c|c|c|c|c|c|c|c|c|c|c|c|c|}
\hline \multirow[b]{2}{*}{ Site } & \multicolumn{3}{|c|}{$\mathrm{CMAQ} \mathrm{D}_{4}$} & \multicolumn{3}{|c|}{ CMAQ D 5} & \multicolumn{3}{|c|}{ CMAQ D 6} & \multicolumn{3}{|c|}{ BETR $D_{5}$} & \multicolumn{3}{|c|}{ DEHM D 5} \\
\hline & Frac. Bias & Frac. Error & Error & Frac. Bias & Frac. Error & Error & Frac. Bias & Frac. Error & Error & Frac. Bias & Frac. Error & Error & Frac. Bias & Frac. Error & Error \\
\hline Bratt's Lake, SK & -0.145 & 0.145 & 0.352 & 1.24 & 1.24 & 6.25 & 0.114 & 0.114 & 0.0376 & 0.383 & 0.383 & 0.900 & -0.303 & 0.303 & 0.500 \\
\hline Whistler, BC & -1.89 & 1.89 & 43.7 & -0.354 & 0.354 & 1.93 & -1.56 & 1.56 & 1.31 & -1.01 & 1.01 & 4.30 & -0.485 & 0.485 & 2.50 \\
\hline Downsview, ON & 0.591 & 0.591 & 9.23 & 0.390 & 0.390 & 26.6 & -0.518 & 0.518 & 2.55 & -1.58 & 1.58 & 48.5 & -0.651 & 0.651 & 27.0 \\
\hline Fraserdale, ON & -1.45 & 1.45 & 4.53 & 0.0164 & 0.0164 & 0.0313 & -1.52 & 1.52 & 0.354 & 0.417 & 0.417 & 1.00 & 0.100 & 0.100 & 0.200 \\
\hline Ucluelet, BC & -1.93 & 1.93 & 43.2 & -0.992 & 0.992 & 4.84 & -1.71 & 1.71 & 1.11 & - & - & - & -1.07 & 1.07 & 5.10 \\
\hline Point Reyes, CA & -0.0195 & 0.0195 & 0.0813 & 0.848 & 0.848 & 9.56 & 0.215 & 0.215 & 0.137 & -1.38 & 1.38 & 5.30 & 0.667 & 0.667 & 6.50 \\
\hline Sydney, FL & 0.613 & 0.613 & 4.77 & -0.674 & 0.674 & 41.3 & -0.753 & 0.753 & 2.19 & -1.71 & 1.71 & 75.5 & -1.38 & 1.38 & 67.0 \\
\hline Groton, CT & 0.969 & 0.969 & 7.32 & -0.745 & 0.745 & 52.1 & -1.45 & 1.45 & 10.1 & - & - & - & -1.15 & 1.15 & 70.0 \\
\hline Mean & -0.407 & 0.950 & 14.1 & -0.0334 & 0.658 & 17.8 & -0.897 & 0.980 & 2.22 & -0.812 & 1.08 & 22.6 & -0.534 & 0.726 & 22.4 \\
\hline Median & -0.0825 & 0.791 & 6.05 & -0.169 & 0.709 & 7.91 & -1.10 & 1.10 & 1.21 & -1.19 & 1.19 & 4.80 & -0.568 & 0.659 & 5.80 \\
\hline
\end{tabular}

$$
\begin{gathered}
\text { Frac. Bias }=\left(\frac{m-o}{\frac{m+o}{2}}\right) \\
\text { Frac. Error }=\left(\frac{|m-o|}{\frac{m+o}{2}}\right)
\end{gathered}
$$

$$
\text { Error }=|m-o|
$$


Section S11: Vertical Concentrations

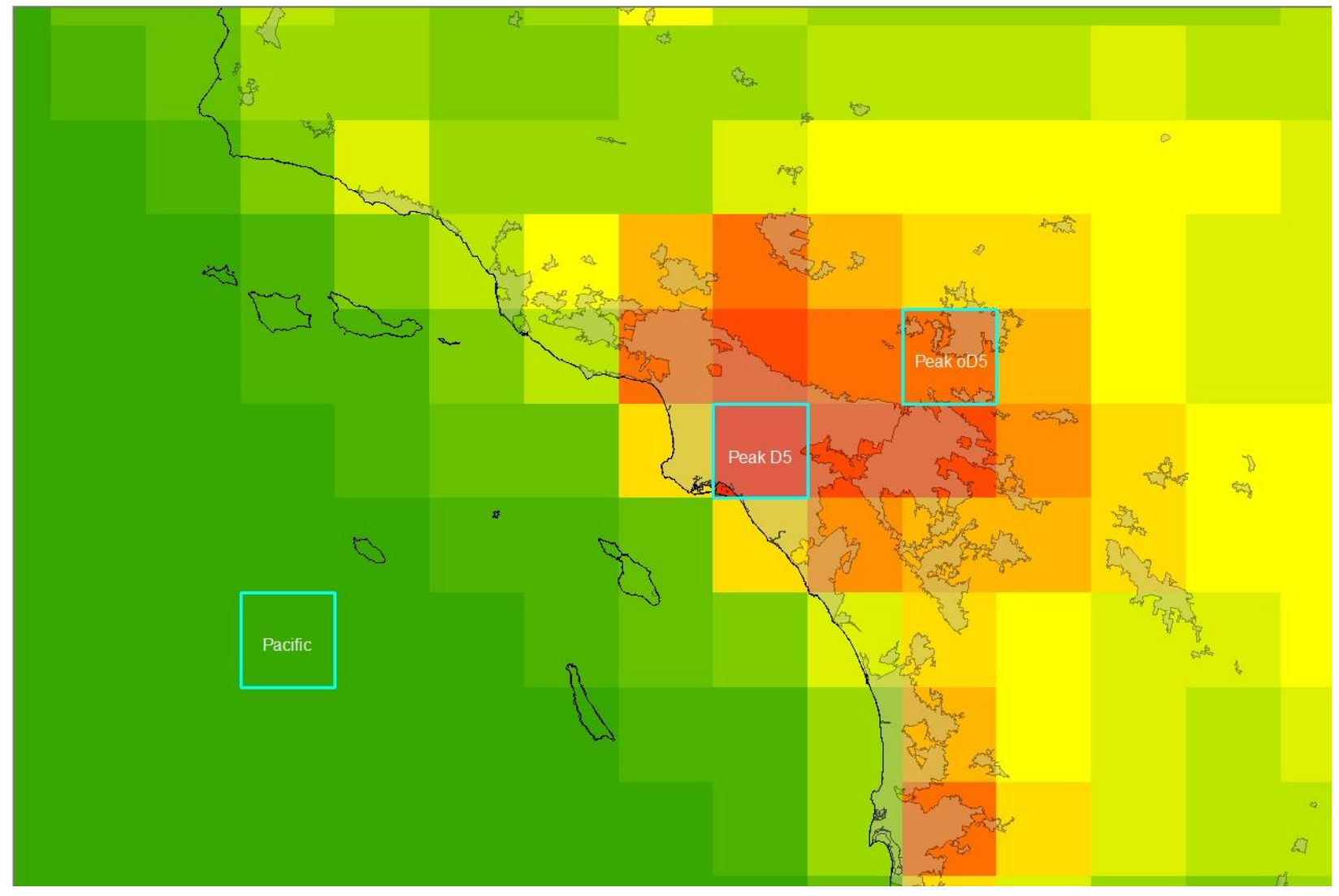

Figure S9: Grid cell locations for vertical analysis in Los Angeles area. 


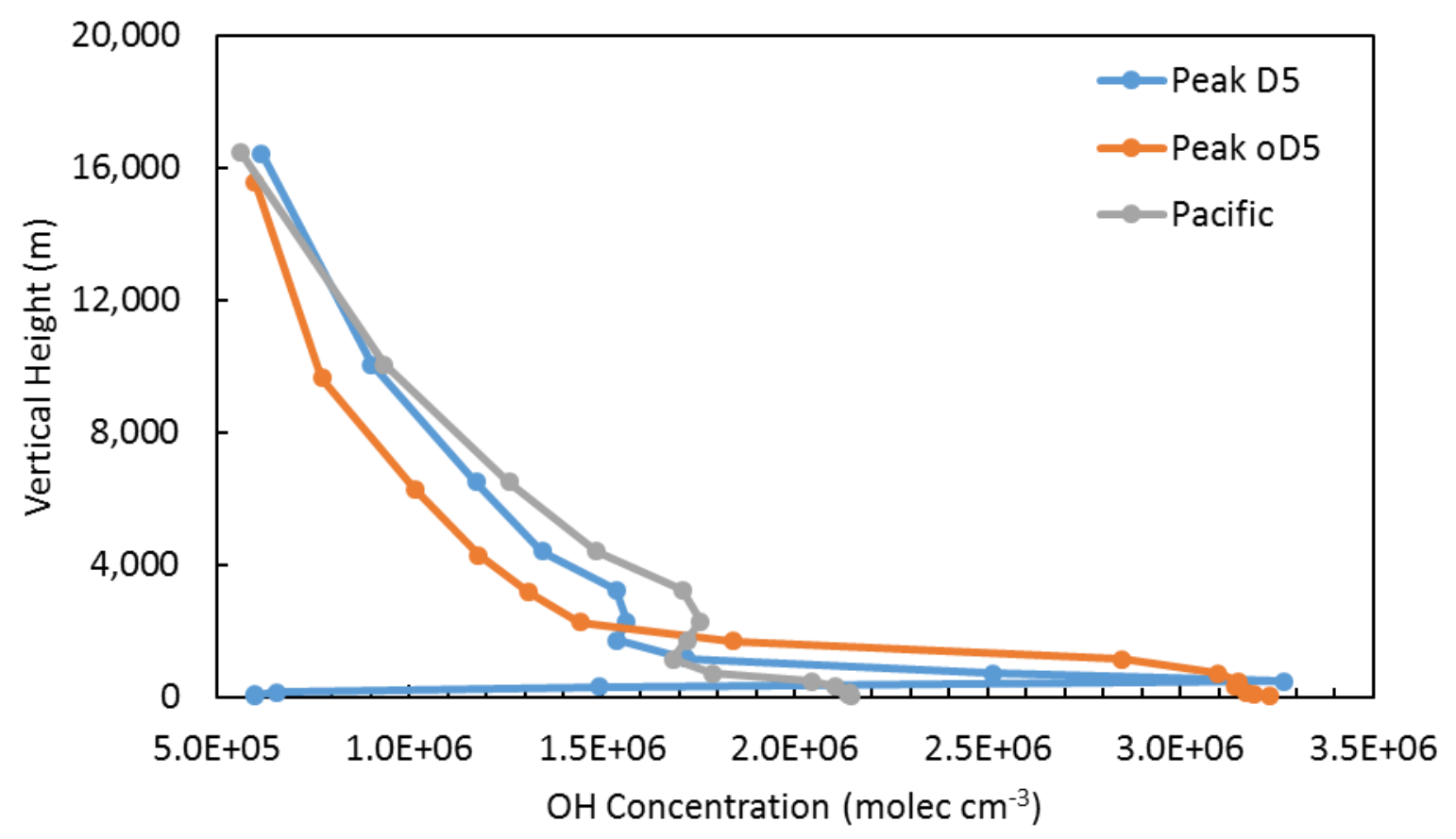

Figure S10: Monthly averaged modeled vertical $\mathrm{OH}$ profiles for grid cells near Los Angeles. Grid cells refer to the location of maximum July $\mathrm{D}_{5}$, maximum July o- $\mathrm{D}_{5}$, and a grid cell over the Pacific Ocean. 


\section{References}

Buser, A. M., Kierkegaard, A., Bogdal, C., MacLeod, M., Scheringer, M., and Hungerbuhler, K.: Concentrations in Ambient Air and Emissions of Cyclic Volatile Methylsiloxanes in Zurich, Switzerland, Environ. Sci. Technol., 47, 7045-7051, doi:10.1021/es3046586, 2013.

Buser, A. M., Bogdal, C., MacLeod, M., and Scheringer, M.: Emissions of decamethylcyclopentasiloxane from Chicago, Chemosphere, 107, 473-475, doi:10.1016/j.chemosphere.2013.12.034, 2014.

Byun, D. W., Pleim, J. E., Tang, R. T., and Bourgeois, A.: Chapter 12: Meteorology-Chemistry Interface Processor (MCIP) for Models-3 Community Multiscale Air Quality (CMAQ) Modeling System, in: Science Algorithms of the EPA Models-3 Community Multiscale Air Quality (CMAQ) Modeling System, United States Environmental Protection Agency, Washington, DC, 1999.

Capela, D., Alves, A., Homem, V., and Santos, L.: From the shop to the drain - Volatile methylsiloxanes in cosmetics and personal care products, Environ. Int., 92-93, 50-62, doi:10.1016/j.envint2016.03.016, 2016.

Dudzina, T., von Goetz, N., Bogdal, C., Biesterbos, J. W. H., and Hungerbuhler, K.: Concentrations of cyclic volatile methylsiloxanes in European cosmetics and personal care products: Prerequisite for human and environmental exposure assessment, Environ. Int., 62, 86-94, doi:10.1016/j.envint.2013.10.002, 2014.

Genualdi, S., Harner, T., Cheng, Y., MacLeod, M., Hansen, K. M., van Egmond, R., Shoeib, M., and Lee, S. C.: Global Distribution of Linear and Cyclic Volatile Methyl Siloxanes in Air, Environ. Sci. Technol., 45, 3349-3354, doi:10.1021/es200301j, 2011.

Hansen, K. M., Christensen, J. H., Brandt, J., Frohn, L. M., Geels, C., Skjoth, C. A., and Li, Y.F.: Modeling short-term variability of alpha-hexachlorocyclohexane in Northern Hemispheric air, J. Geophys. Res.-Atmos., 113, doi:10.1029/2007jd008492, 2008.

Horii, Y., and Kannan, K.: Survey of organosilicone compounds, including cyclic and linear siloxanes, in personal-care and household products, Arch. Environ. Con. Tox., 55, 701-710, doi:10.1007/s00244-008-9172-z, 2008.

McLachlan, M. S., Kierkegaard, A., Hansen, K. M., van Egmond, R., Christensen, J. H., and Skjoth, C. A.: Concentrations and Fate of Decamethylcyclopentasiloxane (D-5) in the Atmosphere, Environ. Sci. Technol., 44, 5365-5370, doi:10.1021/es100411w, 2010.

Navea, J. G., Young, M. A., Xu, S., Grassian, V. H., and Stanier, C. O.: The atmospheric lifetimes and concentrations of cyclic methylsiloxanes octamethylcyclotetrasiloxane $(\mathrm{D}(4))$ and decamethylcyclopentasiloxane $(\mathrm{D}(5))$ and the influence of heterogeneous uptake, Atmos. Environ., 45, 3181-3191, doi:10.1016/j.atmosenv.2011.02.038, 2011.

Roselle, S. J., and Binkowski, F. S.: Chapter 11: Cloud Dynamics and Chemistry, in: Science Algorithms of the EPA Models-3 Community Multiscale Air Quality (CMAQ) Modeling System, United States Environmental Protection Agency, Washington, DC, 1999.

Sander, S. P., Abbatt, J. P. D., Barker, J. R., Burkholder, J. B., Friedl, R. R., Golden, D. M., Huie, R. E., Kolb, C. E., Kurylo, M. J., Moortgat, G. K., Orkin, V. L., and Wine, P. H.: Chemical Kinetics and Photochemical Data for Use in Atmospheric Studies, Evaluation No. 17, Jet Propulsion Laboratory, Pasadena, CA, JPL Publication 10-6, 2011.

Slowik, J. G., Brook, J., Chang, R. Y. W., Evans, G. J., Hayden, K., Jeong, C. H., Li, S. M., Liggio, J., Liu, P. S. K., McGuire, M., Mihele, C., Sjostedt, S., Vlasenko, A., and Abbatt, J. P. D.: Photochemical processing of organic aerosol at nearby continental sites: contrast 
between urban plumes and regional aerosol, Atmos. Chem. Phys., 11, 2991-3006, doi:10.5194/acp-11-2991-2011, 2011.

Spivakovsky, C. M., Logan, J. A., Montzka, S. A., Balkanski, Y. J., Foreman-Fowler, M., Jones, D. B. A., Horowitz, L. W., Fusco, A. C., Brenninkmeijer, C. A. M., Prather, M. J., Wofsy, S. C., and McElroy, M. B.: Three-dimensional climatological distribution of tropospheric OH: Update and evaluation, J. Geophys. Res.-Atmos., 105, 8931-8980, doi:10.1029/1999jd901006, 2000.

Tang, X., Misztal, P. K., Nazaroff, W. W., and Goldstein, A. H.: Siloxanes Are the Most Abundant Volatile Organic Compound Emitted from Engineering Students in a Classroom, Environ. Sci. Technol. Lett., 2, 303-307, doi:10.1021/acs.estlett.5b00256, 2015.

Wang, R., Moody, R. P., Koniecki, D., and Zhu, J.: Low molecular weight cyclic volatile methylsiloxanes in cosmetic products sold in Canada: implication for dermal exposure, Environ. Int., 35, 900-904, doi:10.1016/j.envint.2009.03.009, 2009.

Whelan, M. J., Estrada, E., and van Egmond, R.: A modelling assessment of the atmospheric fate of volatile methyl siloxanes and their reaction products, Chemosphere, 57, 1427-1437, doi:10.1016/j.chemosphere.2004.08.100, 2004.

$\mathrm{Xu}, \mathrm{S}$., and Kropscott, B.: Method for simultaneous determination of partition coefficients for cyclic volatile methylsiloxanes and dimethylsilanediol, Anal. Chem., 84, 1948-1955, doi:10.1021/ac202953t, 2012.

Yucuis, R. A., Stanier, C. O., and Hornbuckle, K. C.: Cyclic siloxanes in air, including identification of high levels in Chicago and distinct diurnal variation, Chemosphere, 92, 905910, doi:10.1016/j.chemosphere.2013.02.051, 2013. 